\title{
Good deal indices in asset pricing: Actuarial and financial implications
}

\author{
Alejandro Balbás*, José Garrido** and Ramin Okhrati*** \\ * University Carlos III of Madrid. C/ Madrid, 126. 28903 Getafe (Madrid, Spain). \\ alejandro.balbas@uc3m.es \\ ** Concordia University. Department of Mathematics and Statistics. 1455 de Maison- \\ neuve Blvd. W., Montreal, Canada H3G 1M8. jose.garrido@concordia.ca \\ *** University of Southampton. Mathematical Sciences. Highfield Southampton SO17 \\ 1BJ, Southampton, UK.r.okhrati@soton.ac.uk
}

A.M.S. 91G10, 91G20, 91B30.

J.E.L. G11, G13, G22.

Keywords Risk measure; Compatibility between prices and risks; Good deal size measurement; Actuarial and financial implications.

Abstract. We integrate into a single optimization problem a risk measure, beyond the variance, and either arbitrage free real market quotations or financial pricing rules generated by an arbitrage free stochastic pricing model. A sequence of investment strategies such that the couple (expected-return,risk) diverges to $(+\infty,-\infty)$ will be called a good deal. The existence of such a sequence is equivalent to the existence of an alternative sequence of strategies such that the couple (risk,price) diverges to $(-\infty,-\infty)$. Moreover, by appropriately adding the riskless asset, every good deal may generate a new one only composed of strategies priced at one.

We will see that good deals often exist in practice, and the main objective of this paper will be to measure the good deal size. The provided good deal indices will equal an optimal ratio between both risk and price, and there will exist alternative interpretations of these indices. They also provide the minimum relative (per dollar) price modification that prevents the existence of good deals. Moreover, they will be a crucial instrument to detect those securities or marketed claims which are over- or under-priced.

Many classical actuarial and financial optimization problems may generate wrong solutions if the used market quotations or stochastic pricing models do not prevent the existence of good deals. This fact is illustrated in the paper, and we point out how the provided good deal indices may be useful to overcome this caveat. Numerical experiments are included as well.

\section{Introduction}

The use of risk functions beyond the variance is becoming more and more frequent in both actuarial and financial studies. Nevertheless, when the most important arbitrage free pricing models of financial economics (binomial, trinomial trees, Black and Scholes, stochastic volatility, etc.) and the most important risk functions (VaR, CVaR, weighted-CVaR, robust-CVaR, spectral measures, 
etc.) are combined in a single problem, one often faces the existence of sequences of investment strategies (good deals, or $G D$ ) whose pairs (expected-return,risk) diverge to $(+\infty,-\infty)$. The existence of $G D$ is equivalent to the existence of alternative sequences of investment strategies whose pairs (risk,price) diverge to $(-\infty,-\infty)$. This pathological finding has been analyzed in Balbás and Balbás (2009) and Balbás et al. (2016a), where explicit examples of the sequences above have been constructed and their performance empirically tested. The main conclusion was that the divergence of (expected-return, risk) to $(+\infty,-\infty)$ is more theoretical than real, but the performance of the constructed $G D$ was good enough. The $G D$ were collections of options providing much better realized Sharpe ratios than their underlying assets.

In this paper we will deal with a couple $(\rho, \Pi)$ composed of the risk measure $\rho$ and the pricing rule $\Pi$. The pair $(\rho, \Pi)$ will be called non compatible if it implies the existence of a $G D$, and the main objective of this paper will be the measurement of the $G D$ size by means of a new index denoted by $\tilde{N}$ or $\tilde{N}(\rho, \Pi)$. An important precedent in financial theory is the notion of arbitrage. Though the absence of arbitrage always holds in theoretical approaches, real market quotations sometimes reflect the existence of arbitrage. For this reason some years ago many authors defined several measures of the arbitrage size. This allowed them to address interesting questions such as pricing and hedging issues under transaction costs, cross-market arbitrage, integration between markets, trading systems, valuation of embedded derivatives, etc. Similarly, the existence of $G D$ (or the lack of compatibility) must be measured now, because in some sense it indicates an important lack of balance between the risk that the investor is facing and the wealth that he/she is expecting. As we will see, these unbalanced situations may lead to wrong decisions in several fields. For instance, managers could pay too high prices or compose inefficient portfolios, and insurers could buy non-optimal reinsurance contracts or receive insufficient premiums.

The arbitrage measurement has been addressed from several perspectives. One of them was related to the capital profits generated by an arbitrage strategy (Balbás et al., 1999). Nevertheless, if the arbitrage strategy can be repeated time and again, the arbitrage profit will be multiplied time and again also, and therefore it will become infinity. To prevent this caveat Balbás et al. (1999) measure the arbitrage level as the maximum ratio between the arbitrage income and the value of the sold assets, i.e., these authors give a relative measure of the arbitrage degree. Similarly, when (risk, price) diverges to $(-\infty,-\infty)$ we will need to maximize the risk/price ratios, otherwise we will face unbounded optimization problems.

A risk/price ratio is an objective function which does not satisfy many desirable analytical properties (continuity, convexity, differentiability, etc.), therefore its optimization can be simplified by dealing instead with vector optimization problems involving both risk and price. Since Harry Markowitz published his seminal results, it is known that multiobjective analyses are useful in many financial topics. In particular, for portfolio selection several interesting approaches exist, such as Ballestero and Romero (1996), Ballestero et al. (2012), Dash and Kajiji (2014), among others. With respect to the simultaneous opti- 
mization of both risk and price, we apply well-known results to optimize "risk" under constraints for "price" (Sawaragi et al., 1985).

The paper outline is as follows. Section 2 sets notations and lists assumptions. The index (or measure) $\tilde{N}(\rho, \Pi)$ is derived in Section 3. The first approach will apply when no theoretical pricing model is considered, and only a finite collection of available securities and their market quotations are involved. The advantage of this approach is clear, since it is sufficient to choose a robust (or ambiguous) risk function $\rho$ for the value of $\tilde{N}$ to be model-independent. Beyond the optimal risk/price ratio, there is a second (or dual) interpretation of $\tilde{N}$ that must be highlighted. $\tilde{N}$ coincides with the minimum relative (per dollar) price modification leading to a $G D$-free market. Moreover, the dual approach permits an investor to identify the over-priced securities (to be sold so as to create a $G D$ ) and the under-priced ones (to be bought). Modifying the prices according to the value of $\tilde{N}$, guarantees the $G D$ absence. A numerical example illustrates all the theoretical findings. In particular, it shows how easily $G D$ 's arise in real markets, how to implement a $G D$, and how prices must be modified.

The second approach replaces real market quotations with pricing rules generated by a complete and arbitrage free stochastic pricing model. Completeness may be relaxed also, as will be indicated. Both the primal (optimal risk/price ratios) and the dual (minimum relative price modifications) interpretations of $\tilde{N}$ still apply, but important differences with respect to the model-independent approach will be also found. Indeed, if the stochastic discount factor $(S D F$, also called pricing kernel, Duffie, 1988) of the pricing rule $\Pi$ is not essentially bounded, and the sub-gradient of the risk function $\rho$ is composed of essentially bounded random variables, then the existence of a $G D$ is guaranteed, and the value of $\tilde{N}(\rho, \Pi)$ will be strictly higher than one. In other words, some marketed claims have a current price which should be modified more than $100 \%$. Otherwise the lack of compatibility will remain. This seems to be an important finding because it shows that some marketed claims will be impossible to price correctly with standard pricing methods. This could explain some empirical caveats affecting the price of several securities, including vanilla options (Bondarenko, 2014). As in the model-independent case, we analyze some important examples. In particular, we present a complete analysis involving the Black and Scholes model and the $C V a R$.

The presence of a $G D$ may provoke irrational solutions in many classical problems involving prices and risk functions. Section 4 is devoted to illustrating it with some particular actuarial examples (optimal reinsurance, premium calculation) and some financial examples (asset allocation, risk management). This section is merely illustrative, we do not claim to fully address the solution of the caveats presented. To do so would lengthen substantially the paper. Beyond the presented examples, the $\tilde{N}$ index could be applied to address topics that were studied years ago by means of the arbitrage measurement, such as market integration, valuation of embedded options, trading systems, etc. Therefore, the $G D$ size measurement may open new research lines in finance, insurance, and fields related to prices, risks and returns. 
The last section gives the main conclusions of the paper.

\section{Preliminaries and Notations}

Consider the probability space $(\Omega, \mathcal{F}, \mathbb{P})$ composed of the set of "states of the world" $\Omega$, the $\sigma$-algebra $\mathcal{F}$ and the probability measure $\mathbb{P}$. Denote by $\mathbb{E}(y)$ the mathematical expectation of every $\mathbb{R}$-valued random variable $y$ defined on $\Omega$. Denote by $L^{2}$ the Hilbert space of random variables $y$ on $\Omega$ such that $\mathbb{E}\left(y^{2}\right)<\infty$, endowed with the inner product $(x, y) \rightarrow \mathbb{E}(x y)$ and the norm $\|y\|_{2}=\left(\mathbb{E}\left(y^{2}\right)\right)^{1 / 2}$. Let $[0, T]$ be a time interval. From an intuitive point of view, one can interpret $y \in L^{2}$ as representing the portfolio pay-off at $T$ for some arbitrary investor (finance), or claims within $[0, T]$ for some arbitrary insurer (insurance). Throughout this paper $y$ will represent the random wealth at $T$, although other interpretations would not modify our main conclusions. If $\rho: L^{2} \longrightarrow \mathbb{R}$ is a risk measure then $\rho(y)$ may be understood as the "risk" associated with the wealth $y$. Let us assume that $\rho$ satisfies a representation theorem in the line with Artzner et al. (1999) or Rockafellar et al. (2006). More precisely, consider the sub-gradient of $\rho$

$$
\Delta_{\rho}=\left\{z \in L^{2} ;-\mathbb{E}(y z) \leq \rho(y), \forall y \in L^{2}\right\} \subset L^{2}
$$

composed of those linear expressions lying lower than $\rho . \Delta_{\rho}$ is convex and weakly-compact (Schaeffer, 1970) and $\rho$ is its envelope, in the sense that

$$
\rho(y)=\operatorname{Max}\left\{-\mathbb{E}(y z) ; z \in \Delta_{\rho}\right\}
$$

holds for every $y \in L^{2}$. Furthermore, we assume also that

$$
\{1\} \subset \Delta_{\rho} \subset\left\{z \in L^{2} ; \mathbb{E}(z)=1\right\}
$$

and

$$
\Delta_{\rho} \subset\left\{z \in L^{2} ; \mathbb{P}(z \geq 0)=1\right\} .
$$

These assumptions are equivalent to the usual properties of norm-continuity, sub-additivity, homogeneity, mean dominance, translation invariance and monotonicity. To sum up, we have the following.

Assumption $1 \rho: L^{2} \longrightarrow \mathbb{R}$ is norm-continuous, sub-additive $\left(\rho\left(y_{1}+y_{2}\right) \leq\right.$ $\rho\left(y_{1}\right)+\rho\left(y_{2}\right)$ if $\left.y_{1}, y_{2} \in L^{2}\right)$, positively homogeneous $\left(\rho(\alpha y)=\alpha \rho(y)\right.$ if $y \in L^{2}$ and $\alpha \geq 0)$, mean dominating $\left(\rho(y) \geq-\mathbb{E}(y)\right.$ if $\left.y \in L^{2}\right)$, translation invariant $\left(\rho(y+k)=\rho(y)-k\right.$ if $y \in L^{2}$ and $\left.k \in \mathbb{R}\right)$ and decreasing $\left(\rho\left(y_{1}\right) \leq \rho\left(y_{2}\right)\right.$ if $y_{1}, y_{2} \in L^{2}$ and $\left.\mathbb{P}\left(y_{1}-y_{2} \geq 0\right)=1\right)$.

Consider a closed sub-space $Y \subset L^{2}$ of reachable pay-offs. It includes many cases. For instance, we can consider that there exists a set $\mathcal{T} \subset[0, T]$ of trading dates, a filtration $\left(\mathcal{F}_{t}\right)_{t \in \mathcal{T}}$ such that $\mathcal{F}_{0}=\{\varnothing, \Omega\}$ and $\mathcal{F}_{T}=\mathcal{F}$, and a $\mathbb{R}^{m+1}$-valued adapted price process $S=\left(S_{0}, S_{1}, \ldots, S_{m}\right)$ such that every $y \in Y$ 
is a marketed claim (or a final wealth replicated by means of a self-financing portfolio adapted to the filtration). As a second example, we can deal with a static approach such that $\mathcal{T}=\{0, T\}$ and $Y$ is a finite-dimensional space generated by $m+1$ securities $\left\{S_{0}, S_{1}, \ldots, S_{m}\right\} \subset L^{2}$ available in the market. Consider also a linear and continuous pricing rule $\Pi: Y \longrightarrow \mathbb{R}$ providing us with the price $\Pi(y)$ of every $y \in Y$ at $t=0$. Under the first framework above $\Pi(y)$ coincides with the initial price of the self-financing portfolio leading to the pay-off $y$ (notice that the absence of arbitrage implies that two adapted and self-financing portfolios leading to the same pay-off will have the same initial price). Under the second framework we can consider that $\Pi(y)$ is just a trivial linear expression of the initial prices of the available assets. We will assume the existence of a riskless asset $(1 \in Y)$ and a null interest rate, i.e.,

$$
\Pi(1)=1 .
$$

Obviously, these assumptions are not at all restrictive. In particular, (5) can be easily achieved by the usual normalization method.

The properties of $\rho$ (Assumption 1) and $\Pi$ allow us to establish Proposition 1 below. We will omit the proof because it is similar to one that can be found in Balbás and Balbás (2009).

Proposition 1 The following statements are equivalent;

a) There exists a sequence $\left(y_{n}\right)_{n=1}^{\infty} \subset Y$ such that $\Pi\left(y_{n}\right) \leq 0, n=1,2, \ldots$ and $\lim _{n \rightarrow \infty} \rho\left(y_{n}\right)=-\infty$.

b) For every $a \in \mathbb{R}$ there exists a sequence $\left(y_{n}\right)_{n=1}^{\infty} \subset Y$ such that $\Pi\left(y_{n}\right) \leq a$, $n=1,2, \ldots$ and $\lim _{n \rightarrow \infty} \rho\left(y_{n}\right)=-\infty$.

c) There exists a sequence $\left(y_{n}\right)_{n=1}^{\infty} \subset Y$ such that $\rho\left(y_{n}\right) \leq 0, n=1,2, \ldots$ and $\lim _{n \rightarrow \infty} \Pi\left(y_{n}\right)=-\infty$.

d) For every $a \in \mathbb{R}$ there exists a sequence $\left(y_{n}\right)_{n=1}^{\infty} \subset Y$ such that $\rho\left(y_{n}\right) \leq a$, $n=1,2, \ldots$ and $\lim _{n \rightarrow \infty} \Pi\left(y_{n}\right)=-\infty$.

e) There exists a sequence $\left(y_{n}\right)_{n=1}^{\infty} \subset Y$ such that $\lim _{n \rightarrow \infty} \rho\left(y_{n}\right)=-\infty$ and $\lim _{n \rightarrow \infty} \Pi\left(y_{n}\right)=-\infty$.

Let us now recall the notion of "compatibility" of Balbás and Balbás (2009).

Definition 2 The couple $(\rho, \Pi)$ is said to be non-compatible if any of $a), b), c)$, d) or e) above hold.

Remark 3 Suppose that $(\rho, \Pi)$ is non-compatible. Consider the sequence

$$
\left(y_{n}\right)_{n=1}^{\infty} \subset Y
$$

of Proposition $1 a$. The price of the sequence $\left(y_{n}-\Pi\left(y_{n}\right)+1\right)_{n=1}^{\infty}$ remains equal to 1 (see (5)), i.e.,

$$
\Pi\left(y_{n}-\Pi\left(y_{n}\right)+1\right)=1, n=1,2, \ldots
$$

The risk function satisfies (see Assumption 1)

$$
\rho\left(y_{n}-\Pi\left(y_{n}\right)+1\right)=\rho\left(y_{n}\right)+\Pi\left(y_{n}\right)-1 \leq \rho\left(y_{n}\right) \rightarrow-\infty .
$$


Bearing in mind that $\rho$ is mean-dominating, the expected value of $y_{n}-\Pi\left(y_{n}\right)+1$ satisfies

$$
\mathbb{E}\left(y_{n}-\Pi\left(y_{n}\right)+1\right) \geq-\rho\left(y_{n}-\Pi\left(y_{n}\right)+1\right) \rightarrow+\infty .
$$

Combining (6), (7) and (8) we have a sequence of investment strategies whose risk goes to minus infinity while its expected return goes to plus infinity.

The "pathology" presented in Remark 3 is not at all strange in asset pricing. As illustrated by Balbás et al. (2016a), the most common arbitrage-free pricing models (Black and Scholes, Heston, etc.) reproduced this anti-intuitive behavior when combined with the most popular coherent risk measures $(C V a R$, weighted $C V a R$, etc.) or the $V a R$ risk measure (despite the fact that $V a R$ does not satisfy Assumption 1). Furthermore, this caveat may also arise if one incorporates ambiguity in the pricing model (i.e., $\mathbb{P}$ is not perfectly known) and deals with robust risk measures (Balbás et al., 2016b). Henceforth, strategies producing the pathology above will be called good deals in this paper.

Definition 4 The sequence $\left(y_{n}\right)_{n=1}^{\infty} \subset Y$ is said to be a $G D$ if all the following conditions hold:

$$
\left\{\begin{array}{l}
\Pi\left(y_{n}\right)=1, \quad n=1,2, \ldots, \\
\mathbb{E}\left(y_{n}\right) \rightarrow+\infty \\
\rho\left(y_{n}\right) \rightarrow-\infty
\end{array}\right.
$$

Remark 5 Keeping in mind Remark 3 , it is obvious that $(\rho, \Pi)$ is compatible if and only if there is no GD.

\section{Good Deal Indices}

A critical assumption in financial theory is the absence of arbitrage in real markets and asset pricing models. Since real market data sometimes exhibit the existence of arbitrage, a major topic in finance 20 years ago was the measurement of the arbitrage size (Prisman, 1986, Davis et al., 1993, Kamara and Miller, 1995, Chen and Knez, 1995, Kempf and Korn, 1998, etc.). This allowed the authors to address several interesting questions such as pricing and hedging issues under transaction costs, cross-market arbitrage, integration between markets, trading systems, etc. Similarly, the existence of a $G D$ (or the lack of compatibility) must be measured, because in some sense it indicates a lack of balance between the risk that the investor is facing and the wealth that he/she is expecting. As we will see, these unbalanced situations may lead to wrong decisions in several fields. For instance, investors could end-up paying over-expensive prices or compose inefficient portfolios, and insurers could end-up buying non-optimal reinsurance contracts or receive insufficient premiums.

Going back to arbitrage measurement, we see that different approaches have been proposed. Some were related to the fundamental theorems of asset pricing (Chen and Knez, 1995), others were justified by means of micro-structure models 
(Kempf and Korn, 1998), etc. The methodology of Balbás et al. (1999) and (2000) was related to the profits generated by the arbitrageur. We will be inspired by this approach in order to measure the $G D$ size, since it will enable a measure in monetary terms.

If an arbitrage strategy is available and we do not impose any constraint, then it is easy to prove that the absolute available arbitrage profit becomes unbounded. For that reason Balbás et al. (1999) measured arbitrage in relative terms, or by mean of ratios. This caveat also applies when measuring the $G D$ size. Indeed, Proposition 1 shows that for negative prices one can construct strategies whose risk goes to minus infinity (Proposition 1a), while for negative risks one can obtain "infinite profits" (Proposition 1c). Hence, we will propose relative measures here as well. More accurately, we will measure with respect to the market value of the sold assets or, equivalently, we will impose a short position lower than one dollar.

Remark 6 Since both $\rho$ and $\Pi$ are positively homogeneous, the existence of a strategy $y \in Y$ such that $\Pi(y) \leq 0$ and $\rho(y)<0$ will imply that $\Pi(\alpha y) \leq 0$ and $\lim _{\alpha \rightarrow+\infty} \rho(a y)=-\infty$, and the caveat of Proposition $1 a$ will hold. Therefore, the fulfillment of the implication

$$
y \in Y, \Pi(y) \leq 0 \quad \Rightarrow \quad-\rho(y) \leq 0
$$

is a necessary and sufficient condition to prevent the existence of a GD.

\subsection{Market data linked indices}

In the first approach we will consider a finite set of available securities

$$
\left\{S_{0}, S_{1}, \ldots, S_{m}\right\} \subset L^{2}
$$

$S_{0}=1$ denoting the riskless asset. We will assume that $\left\{S_{0}, S_{1}, \ldots, S_{m}\right\}$ are linearly independent ${ }^{1}$, and their current prices $p_{0}=1, p_{1}, \ldots, p_{m}$ are observable in the market. In order to prevent some mathematical problems, along with Assumption 1, in this section we impose the following assumption.

Assumption $2 \mathbb{P}\left(S_{j} \geq 0\right)=1, j=1,2, \ldots, m$. Consequently, the absence of arbitrage implies that $p_{j}>0, j=1,2, \ldots, m$.

The closed sub-space $Y \subset L^{2}$ will be the linear manifold generated by the $m+1$ available assets, and the pricing rule $\Pi$ will be the obvious one:

$$
\Pi\left(\sum_{j=0}^{m} y_{j} S_{j}\right)=\sum_{j=0}^{m} y_{j} p_{j} .
$$

\footnotetext{
${ }^{1}$ i.e., there are no non-trivial linear combinations leading to the null asset, or, equivalently, the range of the covariance matrix of $\left\{S_{1}, S_{2}, \ldots, S_{m}\right\}$ equals $m$.
} 
Our index $\tilde{N}\left(\rho,\left(S_{j}\right)_{j=0}^{m},\left(p_{j}\right)_{j=0}^{m}\right)$ of the $G D$ size is defined as the optimal value of the following optimization problem ${ }^{2}$ :

$$
M a x-\rho\left(\sum_{j=0}^{m}\left(x_{j}-y_{j}\right) S_{j}\right)\left\{\begin{array}{l}
\sum_{j=0}^{m} p_{j} y_{j} \leq 1, \\
\sum_{j=0}^{m}\left(x_{j}-y_{j}\right) p_{j} \leq 0, \\
x_{j}, y_{j} \geq 0, \quad j=0,1, \ldots, m,
\end{array}\right.
$$

where $\left(\left(x_{j}\right)_{j=0}^{m},\left(y_{j}\right)_{j=0}^{m}\right) \in \mathbb{R}^{m+1} \times \mathbb{R}^{m+1}$ is the decision variable. The interpretation of (12) is as follows. Every portfolio $x-y=\left(x_{j}-y_{j}\right)_{j=0}^{m}$ is represented by the vector of purchases $x=\left(x_{j}\right)_{j=0}^{m}$ and the vector of sales $y=\left(y_{j}\right)_{j=0}^{m}$. The first constraint imposes a short position lower than 1 dollar (as justified above) and the second one imposes a non-positive global price. Thus, if the desired implication (10) were to hold, then the objective function could not be positive, and the objective maximum value would be reached at $x=y=0$ and would equal $\tilde{N}\left(\rho,\left(S_{j}\right)_{j=0}^{m},\left(p_{j}\right)_{j=0}^{m}\right)=0$. The failure of (10) would lead to a positive value of $\tilde{N}\left(\rho,\left(S_{j}\right)_{j=0}^{m},\left(p_{j}\right)_{j=0}^{m}\right)$. More precisely, we have the following result.

Proposition 7 Problem (12) is feasible, bounded and solvable ${ }^{3}$, with an optimal value $\tilde{N}\left(\rho,\left(S_{j}\right)_{j=0}^{m},\left(p_{j}\right)_{j=0}^{m}\right) \geq 0$. Furthermore, $(\rho, \Pi)$ is compatible (or $G D$ free, Remark 5) if and only if $\tilde{N}\left(\rho,\left(S_{j}\right)_{j=0}^{m},\left(p_{j}\right)_{j=0}^{m}\right)=0$.

Proof. The objective function is obviously continuous (see Assumption 1) and the feasible set is obviously non-void $(x=y=0$ satisfies the problem constraints), bounded and closed (and therefore compact) because every $p_{j}$ is positive. Hence, (12) is solvable due to the Weierstrass Theorem. Since $x=y=0$ is feasible and $\rho(0)=0$, the inequality $\tilde{N}\left(\rho,\left(S_{j}\right)_{j=0}^{m},\left(p_{j}\right)_{j=0}^{m}\right) \geq 0$ becomes obvious.

Suppose that $\tilde{N}\left(\rho,\left(S_{j}\right)_{j=0}^{m},\left(p_{j}\right)_{j=0}^{m}\right)>0$. Then, the solution $\left(x^{*}, y^{*}\right)$ of (12) satisfies

$$
\rho\left(\sum_{j=0}^{m}\left(x_{j}^{*}-y_{j}^{*}\right) S_{j}\right)<0
$$

\footnotetext{
${ }^{2}$ As usual, the optimal value of a maximization (minimization) problem will be the supremum (infimum) of the objective function in the feasible set. This value may become $+\infty$ $(-\infty)$.

${ }^{3}$ An optimization problem will be said to be feasible if the constraints generate a nonvoid feasible set. A feasible minimization (maximization) problem will be said to be bounded if the objective function has a finite infimum (supremum) in the feasible set. A bounded minimization (maximization) problem will be said to be solvable if its infimum (supremum) value is attainable in the feasible set.
} 
and $\sum_{j=0}^{m}\left(x_{j}^{*}-y_{j}^{*}\right) p_{j} \leq 0$, the implication (10) does not hold, and Remark 6 implies that there is a $G D$. Conversely, suppose that $\tilde{N}\left(\rho,\left(S_{j}\right)_{j=0}^{m},\left(p_{j}\right)_{j=0}^{m}\right)=$ 0 and let us see that (10) will hold. If (10) failed then we could take $y \in Y$ with $\Pi(y) \leq 0$ and $-\rho(y)>0 . y$ is a linear combination of $\left\{S_{0}, S_{1}, \ldots, S_{m}\right\}$ so

$$
y=\sum_{j=0}^{m}\left(x_{j}-y_{j}\right) S_{j},
$$

for some $x_{j}, y_{j} \geq 0, j=0,1,2, \ldots, m$. If $\sum_{j=0}^{m} p_{j} y_{j} \leq 1$ then

$$
\tilde{N}\left(\rho,\left(S_{j}\right)_{j=0}^{m},\left(p_{j}\right)_{j=0}^{m}\right) \geq-\rho(y)>0
$$

and we have a contradiction. If $\sum_{j=0}^{m} p_{j} y_{j}>1$ then we could take $x_{j}^{\prime}=$ $x_{j} /\left(\sum_{j=0}^{m} p_{j} y_{j}\right)$ and $y_{j}^{\prime}=y_{j} /\left(\sum_{j=0}^{m} p_{j} y_{j}\right)$, and we would have the same contradiction because $\rho$ is positively homogeneous.

Problem (12) is concave. Bearing in mind Assumption 1, (1), (2), (3) and (4), and proceeding as in Balbás and Balbás (2009) or Balbás et al. (2010), one can prove the existence of a linear dual problem characterizing the solutions of (12). Hence, let us present the result below, omitting the proof as it is similar to the ones in the above cited references.

Theorem 8 Consider the following optimization problem

$$
\operatorname{Min} \lambda \begin{cases}p_{j} \mu-\mathbb{E}\left(S_{j} z\right) \geq 0, & j=0,1, \ldots, m, \\ p_{j}(\mu-\lambda)-\mathbb{E}\left(S_{j} z\right) \leq 0, & j=0,1, \ldots, m, \\ \lambda \geq 0, \mu \geq 0, z \in \Delta_{\rho}, & \end{cases}
$$

where $(\lambda, \mu, z) \in \mathbb{R} \times \mathbb{R} \times \mathrm{L}^{2}$ is the decision variable.

a) Problem (13) is feasible, bounded and solvable, and the optimal values of (12) and (13) coincide, i.e., the maximum value of (12) equals the minimum value of (13).

b) Suppose that $\left(x^{*}, y^{*}\right)$ is (12)-feasible and $\left(\lambda^{*}, \mu^{*}, z^{*}\right)$ is (13)-feasible. Then, $\left(x^{*}, y^{*}\right)$ solves (12) and $\left(\lambda^{*}, \mu^{*}, z^{*}\right)$ solves (13) if and only if the complementary slackness conditions below hold:

$$
\begin{cases}\sum_{j=0}^{m}\left(x_{j}^{*}-y_{j}^{*}\right) \mathbb{E}\left(S_{j} z\right) \geq \sum_{j=0}^{m}\left(x_{j}^{*}-y_{j}^{*}\right) \mathbb{E}\left(S_{j} z^{*}\right), & \forall z \in \Delta_{\rho}, \\ \lambda^{*}\left(1-\sum_{j=0}^{m} p_{j} y_{j}^{*}\right)=0, & \\ \mu^{*}\left(\sum_{j=0}^{m}\left(x_{j}^{*}-y_{j}^{*}\right) p_{j}\right)=0, & j=0,1, \ldots, m, \\ x_{j}^{*}\left(p_{j} \mu^{*}-\mathbb{E}\left(S_{j} z^{*}\right)\right)=0, & j=0,1, \ldots, m . \\ y_{j}^{*}\left(\mathbb{E}\left(S_{j} z^{*}\right)-\left(\mu^{*}-\lambda^{*}\right) p_{j}\right)=0, & \end{cases}
$$


Corollary 9 Consider the following problem

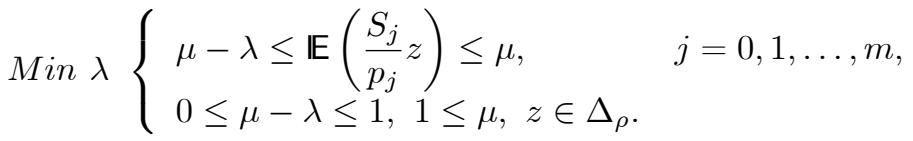

a) Problem (15) is feasible, bounded and solvable, and the optimal values for (12) and (15) coincide.

b) Suppose that $\left(x^{*}, y^{*}\right)$ is (12)-feasible and $\left(\lambda^{*}, \mu^{*}, z^{*}\right)$ is (15)-feasible. Then, $\left(x^{*}, y^{*}\right)$ solves (12) and $\left(\lambda^{*}, \mu^{*}, z^{*}\right)$ solves (15) if and only if the complementary slackness conditions below hold:

$$
\begin{cases}\sum_{j=0}^{m}\left(x_{j}^{*}-y_{j}^{*}\right) \mathbb{E}\left(S_{j} z\right) \geq \sum_{j=0}^{m}\left(x_{j}^{*}-y_{j}^{*}\right) \mathbb{E}\left(S_{j} z^{*}\right), & \forall z \in \Delta_{\rho}, \\ \lambda^{*}\left(1-\sum_{j=0}^{m} p_{j} y_{j}^{*}\right)=0, & \\ \sum_{j=0}^{m} p_{j} x_{j}^{*}=\sum_{j=0}^{m} p_{j} y_{j}^{*} & j=0,1, \ldots, m, \\ x_{j}^{*}\left(\mu^{*}-\mathbb{E}\left(\frac{S_{j}}{p_{j}} z^{*}\right)\right)=0, & j=0,1, \ldots, m . \\ y_{j}^{*}\left(\mathbb{E}\left(\frac{S_{j}}{p_{j}} z^{*}\right)-\left(\mu^{*}-\lambda^{*}\right)\right)=0, & \end{cases}
$$

Proof. Indeed, if $(\lambda, \mu, z)$ is (13)-feasible and $\lambda>\mu$ then $(\mu, \mu, z)$ is feasible too (see (4)) and the objective function decreases, so the constraint $\mu-\lambda \geq 0$ will not be at all restrictive. Besides, (3) along with the first constraint of (13) for $j=0$ trivially lead to $\mu \geq 1$. Moreover, (3) along with the second constraint of (13) for $j=0$ trivially lead to $\mu-\lambda \leq 1$. Lastly, $\mu \geq 1$ implies that the third condition in (14) is equivalent to the third one in (16).

Corollary $10(\rho, \Pi)$ is compatible if and only if there exists $z^{*} \in \Delta_{\rho}$ such that $\mathbb{E}\left(\frac{S_{j}}{p_{j}} z^{*}\right)=1$, for $j=0,1,2, \ldots, m$.

Proof. Indeed, if $(\rho, \Pi)$ is compatible then Proposition 7 shows that the solution $\left(\lambda^{*}, \mu^{*}, z^{*}\right)$ of (15) satisfies $\lambda^{*}=0$. Thus, the constraints of (15) imply that $\mathbb{E}\left(\frac{S_{j}}{p_{j}} z^{*}\right)=\mu^{*}$, for $j=0,1,2, \ldots, m$. In particular, for $j=0$ we have (see (3)) $1=\mathbb{E}\left(z^{*}\right)=\mu^{*}$.

Conversely, suppose that the existence of $z^{*} \in \Delta_{\rho}$ holds. Then, take $\left(x^{*}, y^{*}\right)=$ $(0,0)$ and $\left(\lambda^{*}, \mu^{*}, z^{*}\right)=\left(0,1, z^{*}\right)$, and it is easy to verify that they are feasible and satisfy (16), so the optimal value of (15) will become

$$
\tilde{N}\left(\rho,\left(S_{j}\right)_{j=0}^{m},\left(p_{j}\right)_{j=0}^{m}\right)=\lambda^{*}=0
$$


and Proposition 7 shows that $(\rho, \Pi)$ is compatible.

If $(\rho, \Pi)$ is not compatible one could try to modify $\left(p_{j}\right)_{j=0}^{m}$ so as to recover compatibility. According to Corollary 9 , if $\left(\lambda^{*}, \mu^{*}, z^{*}\right)$ is the solution of (15), $p_{j}^{*}=\mathbb{E}\left(S_{j} z^{*}\right)$, for $j=0,1,2, \ldots, m$ could be a good alternative. Next, let us show that, in some sense, this is "the best alternative", since it minimizes the maximum relative (or per dollar) price modification.

Corollary 11 Consider a dual solution $\left(\lambda^{*}, \mu^{*}, z^{*}\right)$ and take $p_{j}^{*}=\mathbb{E}\left(S_{j} z^{*}\right)$, for $j=0,1,2, \ldots, m$. Suppose that ${ }^{4}$

$$
p_{j}^{*}>0, j=0,1, \ldots, m .
$$

Then:

a) $p_{0}^{*}=1$.

b) $\tilde{N}\left(\rho,\left(S_{j}\right)_{j=0}^{m},\left(p_{j}^{*}\right)_{j=0}^{m}\right)=0$, i.e., if $\Pi^{*}$ is given by $\Pi^{*}\left(\sum_{j=0}^{m} y_{j} S_{j}\right)=$ $\sum_{j=0}^{m} y_{j} p_{j}^{*}$ then $\left(\rho, \Pi^{*}\right)$ is compatible.

c)

$$
\tilde{N}\left(\rho,\left(S_{j}\right)_{j=0}^{m},\left(p_{j}\right)_{j=0}^{m}\right)=\operatorname{Max}\left\{\frac{p_{j}^{*}}{p_{j}}-\frac{p_{i}^{*}}{p_{i}} ; i, j=0,1, \ldots, m\right\} .
$$

In particular, $\left(p_{j}^{*}\right)_{j=0}^{m}=\left(p_{j}\right)_{j=0}^{m}$ if and only if $(\rho, \Pi)$ is compatible.

d) Consider an arbitrary $\left(p_{j}^{* *}\right)_{j=0}^{m} \in \mathbb{R}^{m+1}$. If $p_{0}^{* *}=1, p_{j}^{* *}>0$, for $j=$ $0,1, \ldots, m$, and $\tilde{N}\left(\rho,\left(S_{j}\right)_{j=0}^{m},\left(p_{j}^{* *}\right)_{j=0}^{m}\right)=0$, then

$$
\tilde{N}\left(\rho,\left(S_{j}\right)_{j=0}^{m},\left(p_{j}\right)_{j=0}^{m}\right) \leq \operatorname{Max}\left\{\frac{p_{j}^{* *}}{p_{j}}-\frac{p_{i}^{* *}}{p_{i}} ; i, j=0,1, \ldots, m\right\} .
$$

Proof. a) It trivially follows from (3).

b) It trivially follows from Corollary 10.

c) As in the proof of Corollary 10, if $\tilde{N}\left(\rho,\left(S_{j}\right)_{j=0}^{m},\left(p_{j}\right)_{j=0}^{m}\right)=0$ then $p_{j}=\mathbb{E}\left(S_{j} z^{*}\right)=p_{j}^{*}$, for $j=0,1,2, \ldots, m$, and therefore the right hand side of (18) equals zero too. Suppose that $\tilde{N}\left(\rho,\left(S_{j}\right)_{j=0}^{m},\left(p_{j}\right)_{j=0}^{m}\right)>0$. Consider the solutions $\left(x^{*}, y^{*}\right)$ and $\left(\lambda^{*}, \mu^{*}, z^{*}\right)$ of (12) and (15). Obviously, $\left(x^{*}, y^{*}\right) \neq(0,0)$, and the second and third conditions of (16) imply that $x^{*} \neq 0$ and $y^{*} \neq 0$. If $x_{j_{1}}^{*}>0$ and $y_{j_{2}}^{*}>0$ then (16) implies that

$$
\mathbb{E}\left(\frac{S_{j_{1}}}{p_{j_{1}}} z^{*}\right)=\mu^{*}, \quad \mathbb{E}\left(\frac{S_{j_{2}}}{p_{j_{2}}} z^{*}\right)=\left(\mu^{*}-\lambda^{*}\right) .
$$

\footnotetext{
${ }^{4}(17)$ will hold if $\mathbb{P}\left(z^{*}>0\right)=1$. Analogously, if Assumption 2 is replaced by the stronger property $\mathbf{P}\left(S_{j}>0\right)=1$, for $j=1,2, \ldots, m$, then (17) will hold because $\mathbb{P}\left(z^{*} \geq 0\right)=1$ due to (4) and $z^{*} \neq 0$ due to (3). Lastly, bearing in mind the constraints of (15), (17) will also hold if $\mu^{*}-\lambda^{*}>0$.
} 
Hence,

$$
\frac{p_{j_{1}}^{*}}{p_{j_{1}}}-\frac{p_{j_{2}}^{*}}{p_{j_{2}}}=\mu^{*}-\left(\mu^{*}-\lambda^{*}\right)=\lambda^{*} .
$$

For an arbitrary couple $(i, j)$, and keeping in mind the constraints of (15), we have that

$$
\frac{p_{j}^{*}}{p_{j}}-\frac{p_{i}^{*}}{p_{i}} \leq \mu^{*}-\left(\mu^{*}-\lambda^{*}\right)=\lambda^{*}
$$

Thus, the right hand side of (18) equals $\lambda^{*}$. Hence the result becomes obvious because $\tilde{N}\left(\rho,\left(S_{j}\right)_{j=0}^{m},\left(p_{j}\right)_{j=0}^{m}\right)=\lambda^{*}$, owing to Corollary $9 a$.

d) Corollary 10 implies the existence of $z^{* *} \in \Delta_{\rho}$ such that $p_{j}^{* *}=\mathbb{E}\left(S_{j} z^{* *}\right)$, for $j=0,1,2, \ldots, m$. It is obvious that

$$
\begin{aligned}
& \mu^{* *}=\operatorname{Max}\left\{\mathbb{E}\left(\frac{S_{j}}{p_{j}} z^{* *}\right) ; j=0,1, \ldots, m\right\}=\operatorname{Max}\left\{\frac{p_{j}^{* *}}{p_{j}} ; j=0,1, \ldots, m\right\} \\
& \mu^{* *}-\lambda^{* *}=\operatorname{Min}\left\{\mathbb{E}\left(\frac{S_{j}}{p_{j}} z^{* *}\right) ; j=0,1, \ldots, m\right\}=\operatorname{Min}\left\{\frac{p_{j}^{* *}}{p_{j}} ; j=0,1, \ldots, m\right\}
\end{aligned}
$$

make $\left(\lambda^{* *}, \mu^{* *}, z^{* *}\right)(15)$-feasible. Therefore, $\tilde{N}\left(\rho,\left(S_{j}\right)_{j=0}^{m},\left(p_{j}\right)_{j=0}^{m}\right)=\lambda^{*} \leq$ $\lambda^{* *}=\mu^{* *}-\left(\mu^{* *}-\lambda^{* *}\right)$, i.e.,

$$
\begin{aligned}
& \tilde{N}\left(\rho,\left(S_{j}\right)_{j=0}^{m},\left(p_{j}\right)_{j=0}^{m}\right) \leq \\
& \operatorname{Max}\left\{\frac{p_{j}^{* *}}{p_{j}} ; j=0,1, \ldots, m\right\}-\operatorname{Min}\left\{\frac{p_{j}^{* *}}{p_{j}} ; j=0,1, \ldots, m\right\} \\
& =\operatorname{Max}\left\{\frac{p_{j}^{* *}}{p_{j}}-\frac{p_{i}^{* *}}{p_{i}} ; i, j=0,1, \ldots, m\right\}
\end{aligned}
$$

Remark 12 Corollary 11 may be interpreted in terms of "fair prices". Indeed, denote by $\left(\lambda^{*}, \mu^{*}, z^{*}\right)$ a solution of $(15)$. If $(\rho, \Pi)$ is non compatible then one can build portfolios with negative risk and zero or negative prices (Proposition 1). According to (16), this is possible if one precisely buys those securities such that $\mathbb{E}\left(\frac{S_{j}}{p_{j}} z^{*}\right)=\mu^{*}$ and sells those satisfying $\mathbb{E}\left(\frac{S_{j}}{p_{j}} z^{*}\right)=\mu^{*}-\lambda^{*}$. In other words, according to the risk measure $\rho$, if $\mathbb{E}\left(\frac{S_{j}}{p_{j}} z^{*}\right)=\mu^{*}\left(\mathbb{E}\left(\frac{S_{j}}{p_{j}} z^{*}\right)=\right.$ $\mu^{*}-\lambda^{*}$ ) then $S_{j}$ is under-priced (over-priced), and, according to Corollary 11, the new prices $p_{j}^{*}=\mathbb{E}\left(S_{j} z^{*}\right)$, for $j=0,1,2, \ldots, m$ will provide us with the lowest relative modification leading to "fair prices" (or GD-free prices). Note that $p_{j}^{*}=\mathbb{E}\left(S_{j} z^{*}\right)=\mu^{*} p_{j} \geq p_{j}$ if $x_{j}^{*}>0\left(p_{j}^{*}=\mathbb{E}\left(S_{j} z^{*}\right)=\left(\mu^{*}-\lambda^{*}\right) p_{j} \leq p_{j}\right.$ if $\left.y_{j}^{*}>0\right)$. 


\subsection{Numerical experiment}

Let us illustrate the results of Section 3.1 with a very simple example. We deal with an arbitrage free and almost model-independent option market; we will see that some premiums must decrease more than $0.4 \%$ in order to prevent the existence for a $G D$. Furthermore, the $G D$ is static, which means that once it is implemented, the portfolio does not have to be rebalanced before the options maturity ${ }^{5}$.

As above, suppose that $S_{0}=1$ is a riskless asset and consider a security $S_{1}$ whose behavior is given by a geometric Brownian motion $(G B M)$ with a current price of 1 , a drift of $1 \%$ and and volatility of $60 \%$. Consider also a derivative market where European calls can be traded. The unique maturity is $1 / 4$ years (three months), and the available strikes are $\{0.82 ; 0.84 ; 0.86 ; \ldots ; 1.4\}$, i.e., the lowest one equals 0.82 , the highest one equals 1.4 , and the increment between two consecutive strikes equals 0.02 . Globally, there are 32 available securities (the riskless asset, the underlying asset and 30 European calls). Suppose that the Black and Scholes model perfectly fits the data, i.e., all of the market prices equal the theoretical ones given by Black and Scholes formula. Accordingly, these are given $b y^{6}$ :

$\left(\begin{array}{ccccc}0.221151109 ; & 0.207527141 ; & 0.194479893 ; & 0.182013559 ; & 0.170128799 \\ 0.158822968 ; & 0.14809037 ; & 0.137922549 ; & 0.12830858 ; & 0.119235385 \\ 0.110688033 ; & 0.102650044 ; & 0.095103673 ; & 0.088030189 ; & 0.08141012 \\ 0.075223495 ; & 0.069450051 ; & 0.064069422 ; & 0.059061311 ; & 0.054405635 \\ 0.050082646 ; & 0.046073045 ; & 0.042358062 ; & 0.038919533 ; & 0.035739953 \\ 0.032802518 ; & 0.030091156 ; & 0.027590546 ; & 0.025286127 ; & 0.023164098\end{array}\right)$.

Since the Black and Scholes model is arbitrage free, this market is arbitrage free as well. Consider an investor who is interested in verifying the compatibility between prices above and the $C V a R_{\alpha}$ risk measure, $\alpha$ being the level of confidence. Suppose that $\alpha=79 \%$. Despite the fact that this investor can verify that the quotations above lead to a constant implied volatility $\sigma=0.6$, and therefore the data confirm in this case the Black and Scholes model, let us assume that he/she is still very ambiguous with respect to that. Accordingly, he/she will accept deviations between the predictions of the log-normal distribution and the realized value of $S_{1}$ in three months. He/she considers that the error between the probabilities of the log-normal distribution and the real probabilities may become $100 \%$. In other words, for every Borel subset $B \subset \mathbb{R}$, the real probability of the event $S_{1} \in B$ will be laying within the spread $\left[0,2 \mathbb{P}\left(S_{1} \in B\right)\right]$, where $\mathbb{P}\left(S_{1} \in B\right)$ is the theoretical probability under log-normality. In such a case, instead the $C V a R_{79 \%}$ risk measure, the investor will use the robust $C V a R_{79 \%}$

\footnotetext{
${ }^{5}$ According to the empirical evidence, the available theoretical arbitrage free pricing models have many problems to match real market prices in active and liquid derivative markets (Bondarenko, 2014). Perhaps, the theoretical models should prevent the existence of a $G D$ as well.

${ }^{6}$ The given matrix provides us with the price of the 30 available European calls. Obviously, the call price decreases as the call strike increases.
} 
(denoted $\left.R C V a R_{79 \%}\right)$. In general,

$$
\operatorname{RCVaR}_{\alpha}(y):=\operatorname{Max}\left\{\operatorname{CVaR}_{(Q, \alpha)}(y) ; 0 \leq \frac{d Q}{d \mathbb{P}} \leq 2\right\},
$$

where $Q$ is a $\mathbb{P}$-continuous probability measure and $C V a R_{(Q, \alpha)}(y)$ is the $C V a R_{\alpha}$ of $y$ under $Q$. Balbás et al. (2016b) shows that the $R C V a R_{\alpha}(y)$ above is well defined for every $y \in L^{2}$, along with the fulfillment of Assumption 1. Moreover the sub-gradient (1) is given by

$$
\left\{z \in L^{2} ; 0 \leq \frac{d Q}{d \mathbb{P}} \leq 2,0 \leq z \leq \frac{1}{1-\alpha}\left(\frac{d Q}{d \mathbb{P}}\right), \mathbb{E}(z)=1\right\} .
$$

It is easy to see that the set above coincides with

$$
\left\{z \in L^{2} ; 0 \leq z \leq \frac{1}{1-(1+\alpha) / 2}, \mathbb{E}(z)=1\right\} .
$$

Since this is the sub-gradient of the $C V a R_{(1+\alpha) / 2}$ risk measure (Rockafellar et $a l ., 2006), R C V a R_{\alpha}=C V a R_{(1+\alpha) / 2}$ and the high ambiguity level of this example only implies that the level of confidence must be increased appropriately. In particular, for $\alpha=79 \%$ one has $(1+\alpha) / 2=89.5 \%$, and our investor will verify the compatibility between the given market and the $C V a R_{89.5 \%}$ risk measure.

The equality $R C V a R_{79 \%}=C V a R_{89.5 \%}$ implies that the existence of ambiguity only forces a larger level of confidence. Nevertheless, it is important to point out that we are dealing with an ambiguous setting. (19) leads to

$$
C V a R_{89.5 \%}(y)=R C V a R_{79 \%}(y)=\operatorname{Max}\left\{\operatorname{CVaR}_{(Q, 79 \%)}(y) ; 0 \leq \frac{d Q}{d \mathbb{P}} \leq 2\right\},
$$

and therefore the $G D$ existence for $C V a R_{89.5 \%}$ will imply the $G D$ existence for every $C V a R_{(Q, 79 \%)}$. With the confidence level $\alpha=79 \%$ the $G D$ existence does not depend on the probability measure $Q$, or, in other words, every found $G D$ will be still a $G D$ if one makes errors when estimating the probabilities. In this sense, the $G D$ is model-independent, and will also satisfy Definition 4 for models beyond the Black and Scholes one.

In order to verify the existence of $G D$, we can solve the linear Problem (15), with $\Delta_{\rho}$ given by (21) for $\alpha=79 \%$ (see Anderson and Nash, 1987). The optimal value becomes

$$
\tilde{N}\left(\rho,\left(S_{j}\right)_{j=0}^{m},\left(p_{j}\right)_{j=0}^{m}\right)=0.004203112 \approx 0.42 \%
$$

and the existence of a $G D$ (or the lack of compatibility, Remark 5) is implied by Proposition 7 .

Once the lack of compatibility is confirmed, (16) enables us to give an explicit $G D$ and the list of under-priced (over-priced) securities. In fact, it is easy to 
check that this list here is:

$$
\left(\begin{array}{l}
\text { Assets_Sold_by_the_GD } \\
\text { Call_Strike_0.96 } \\
\text { Call_Strike_1.16 } \\
\text { Call_Strike_1.3 } \\
\text { Call_Strike_1.36 }
\end{array}\right)\left(\begin{array}{l}
\text { Assets_Bought_- } \\
\text { by_the_GD } \\
\text { Riskless_Asset } \\
\text { Call_Strike_1 } \\
\text { Call_Strike_1.06 } \\
\text { Call_Strike_1.2 } \\
\text { Call_Strike_1.28 } \\
\text { Call_Strike_1.34 } \\
\text { Call_Strike_1.38 } \\
\text { Call_Strike_1.4 }
\end{array}\right) .
$$

Accordingly, and bearing in mind that every modification of prices preventing the $G D$ will conserve the same riskless rate (Corollary 11), the over-priced securities are the European calls with strikes 0.96, 1.16, 1.3 and 1.36, while the calls with strikes $1,1.06,1.2,1.28,1.34,1.38$ and 1.4 are under-priced (Remark 12). The solution of (15) gives $\mu^{*}=1$ and $\mu^{*}-\lambda^{*}=0.995796888$, so (16), Corollary 11 and Remark 12 allow us to implement the minimum relative modification of prices preventing the $G D$ existence. The price of the seven under-priced calls should remain the same $\left(\mu^{*}=1\right)$, while the price of the four over-priced calls should be multiplied by $0.9958\left(\mu^{*}-\lambda^{*} \approx 0.9958\right)$. Thus, in this example $\tilde{N}\left(\rho,\left(S_{j}\right)_{j=0}^{m},\left(p_{j}\right)_{j=0}^{m}\right)$ gives the relative price variation of the expensive assets (see (22)). Once $z^{*}$ is known, the rest of prices should decrease according to the results of Corollary 11. We do not address this straightforward modification here in order to shorten the exposition ${ }^{7}$.

\footnotetext{
${ }^{7}$ The dual solution $z^{*}$ is an exotic derivative of $S_{1}$, namely,

$z^{*}= \begin{cases}9.523809524, & 0.732390081<S_{1}<0.756345122 \\ 9.523809524, & 0.771634941<S_{1}<0.786508847 \\ 9.523809524, & 0.947638208<S_{1}<0.962000836 \\ 6.0890897, & 1.006619358<S_{1}<1.022152267 \\ 9.523809524, & 1.071497097<S_{1}<1.089092088 \\ 1.228932079, & 1.089092088<S_{1}<1.107403466 \\ 9.091953283, & 1.157082562<S_{1}<1.178912787 \\ 5.882406772, & 1.227117704<S_{1}<1.25412988 \\ 3.889725707, & 1.316447058<S_{1}<1.353379522 \\ 2.694707294, & 1.373837141<S_{1}<1.420023944 \\ 2.634172493, & 1.420023944<S_{1}<1.446520681 \\ 2.732181867, & 1.446520681<S_{1}<1.509427691 \\ 0.923565011, & 1.509427691<S_{1}<1.54799097 \\ 2.472733554, & 1.54799097<S_{1}<1.593797149 \\ 5.22380624, & 1.593797149<S_{1}<1.650491631 \\ 5.386339925, & 1.837722647<S_{1}<2.085596933 \\ 0, & \text { Otherwise }\end{cases}$
}




\subsection{Pricing model linked indices}

The approach of Section 3.1 has interesting advantages because it applies to real market data and one does not have to impose any assumptions beyond the absence of arbitrage. Nevertheless, there are also some drawbacks. Indeed, the example of Section 3.2 led to two sets of under and over-priced securities, respectively (see (23)). Suppose that we add a new call option (new strike and same maturity) and the number of available assets becomes 33. Further assume that the new option is again priced according to Black and Scholes formula. Lastly suppose that we compute the new value of the $G D$-index $\tilde{N}$, along with the sets of under and overpriced securities. Can we guarantee that the equality $\tilde{N} \approx 0.42 \%$ and the conclusion of (23) will still hold? We will see that the answer is "no" (Theorem 19), which means that the given index does not reflect "a global maximum $G D$-size for the Black and Scholes model". The given index is model-free and based only on the available market quotations, but it would also be interesting to give an index for the Black and Scholes pricing model itself.

We provide a new $G D$-index for complete pricing models, i.e., cases such that $Y=L^{2}$. Interesting examples are, among others, the binomial model and the Black and Scholes model. If the model is incomplete we can often assume that there is an extension of $\Pi$ to the whole space $L^{2}$ which still prevents the absence of arbitrage. This extension, still denoted by $\Pi$, implies that such incomplete cases also fit in our general framework. The existence of the extension holds, for instance, if the set $\Omega$ only contains finitely many states (Harrison and Kreps, 1979). Therefore, cases such as the usual trinomial models are also included in our analysis. If $\Omega$ contains infinitely many states then the existence of $\Pi$ is also possible. For instance, though "formally" stochastic volatility models are incomplete, in practice the existence of volatility dependent assets is assumed, making them complete. Otherwise it would be impossible to use these models so as to give a unique price of the usual derivatives. Further details about the existence of $\Pi$ under general conditions for $\Omega$ may be found in Luenberger (2001).

The Riesz representation theorem (Schaeffer, 1970) implies the existence of a unique $z_{\Pi} \in L^{2}$ such that

$$
\Pi(y)=\mathbb{E}\left(y z_{\Pi}\right)
$$

holds for every $y \in L^{2}$. Usually $z_{\Pi}$ is called $S D F$, and it must satisfy

$$
\mathbb{P}\left(z_{\Pi}>0\right)=1
$$

in order to prevent arbitrage (Duffie, 1988). Furthermore, (24) and (5) trivially imply that

$$
\mathbb{E}\left(z_{\Pi}\right)=1 \text {. }
$$

In order to prevent some mathematical problems, along with Assumption 1, in Sections 3.3 and 3.4 we impose here also the following Assumption 3. 
Assumption 3 There exists $(\mu, z) \in \mathbb{R} \times \Delta_{\rho}$ such that $\mathbb{P}\left(z \leq \mu z_{\Pi}\right)=1$.

Assumption 3 frequently holds in practice. For instance, it holds if $\rho$ is $C V a R$ or weighted $-C V a R$ and $z_{\Pi}$ is the $S D F$ of the binomial model, the Black and Scholes model, or the standard complete extension of many trinomial models or many stochastic volatility models. Though it is difficult to give an economic interpretation of this technical assumption, the existence of the required $(\mu, z)$ significantly shortens the rest of the exposition. If $(\mu, z)$ did not exist then $(28)$ could be unfeasible, and Theorem 13 would become much more complex. As explained above, since the existence of $(\mu, z)$ holds for the most important risk measures and pricing models, we have chosen to impose Assumption 3 in order to simplify the study.

The new index $\tilde{N}(\rho, \Pi)$ of the $G D$ size is the optimal value of the optimization problem

$$
M a x-\rho(x-y)\left\{\begin{array}{l}
\Pi(y) \leq 1, \\
\Pi(x-y) \leq 0, \\
x, y \in L^{2}, x, y \geq 0 .
\end{array}\right.
$$

Obviously, $(27)$ is always feasible and $\tilde{N}(\rho, \Pi) \geq 0$ because $(x, y)=(0,0)$ satisfies the required constraints. Next, let us give a main result whose proof is similar to those of Theorem 8 and Corollary 9.

Theorem 13 Consider the following problem:

$$
\operatorname{Min} \lambda\left\{\begin{array}{l}
(\mu-\lambda) z_{\Pi} \leq z \leq \mu z_{\Pi}, \\
0 \leq \mu-\lambda \leq 1,1 \leq \mu, z \in \Delta_{\rho},
\end{array}\right.
$$

where $(\lambda, \mu, z) \in \mathbb{R} \times \mathbb{R} \times \mathrm{L}^{2}$ is the decision variable.

a) Problem (28) is feasible, bounded and solvable, and the optimal values of (27) and (28) coincide.

b) Suppose that $\left(x^{*}, y^{*}\right)$ is (27)-feasible and $\left(\lambda^{*}, \mu^{*}, z^{*}\right)$ is (28)-feasible. Then, $\left(x^{*}, y^{*}\right)$ solves (27) and $\left(\lambda^{*}, \mu^{*}, z^{*}\right)$ solves (28) if and only if the following complementary slackness conditions hold:

$$
\left\{\begin{array}{l}
\mathbb{E}\left(\left(x^{*}-y^{*}\right) z\right) \geq \mathbb{E}\left(\left(x^{*}-y^{*}\right) z^{*}\right), \quad \forall z \in \Delta_{\rho} \\
\lambda^{*}\left(1-\mathbb{E}\left(y^{*} z_{\Pi}\right)\right)=0 \\
\mathbb{E}\left(\left(x^{*}-y^{*}\right) z_{\Pi}\right)=0 \\
x^{*}\left(\mu^{*} z_{\Pi}-z^{*}\right)=0 \\
y^{*}\left(z^{*}-\left(\mu^{*}-\lambda^{*}\right) z_{\Pi}\right)=0
\end{array}\right.
$$

Corollary 14 The statements below are equivalent:

a) $(\rho, \Pi)$ is compatible.

b) $\tilde{N}(\rho, \Pi)=0$.

c) $\left(x^{*}, y^{*}\right)=(0,0)$ solves (27).

d) The solution $\left(\lambda^{*}, \mu^{*}, z^{*}\right)$ of (28) satisfies $\lambda^{*}=0$.

e) The solution $\left(\lambda^{*}, \mu^{*}, z^{*}\right)$ of (28) satisfies $\mu^{*}=1$.

$f)$ The solution $\left(\lambda^{*}, \mu^{*}, z^{*}\right)$ of (28) satisfies $z^{*}=z_{\Pi}$. 
Proof. $a) \Rightarrow b)$ If $(\rho, \Pi)$ is compatible then Implication (10) holds. Thus, if $(x, y)$ is $(27)$-feasible the second problem constraint implies that $-\rho(x-y) \leq 0$. Since $(0,0)$ is $(27)$-feasible, $b)$ becomes obvious.

$b) \Rightarrow c)(0,0)$ is $(27)$-feasible, and $-\rho(0) \geq 0$, so $(0,0)$ solves $(27)$ when the optimal objective equals 0 .

c) $\Rightarrow d)$ If $\left(x^{*}, y^{*}\right)=(0,0)$ solves $(27)$ then the optimal value of $(28)$ vanishes.

$d) \Rightarrow e$ ) If the optimal value of (28) vanishes, then the first constraint leads to $z^{*}=\mu^{*} z_{\Pi}$. Taking expectations, and keeping in mind (3) and (26), we have that $\mu^{*}=1$.

$e) \Rightarrow f$ ) If $\mu^{*}=1$, then the first constraint of (28) implies that $z^{*} \leq z_{\Pi}$. Since both random variables have the same expectation (see (3) and (26)), we have that $z^{*}=z_{\Pi}$.

$f) \Rightarrow a)$ Suppose that $z^{*}=z_{\Pi}$. It is very easy to verify that $\left(x^{*}, y^{*}\right)=(0,0)$ and $\left(\lambda^{*}, \mu^{*}, z^{*}\right)=\left(0,1, z_{\Pi}\right)$ are feasible and satisfy $(29)$. If $\left(x^{*}, y^{*}\right)=(0,0)$ solves (27), then (10) must hold, and therefore $(\rho, \Pi)$ must be compatible (see Remark 6). Indeed, if (10) fails because $\Pi(y) \leq 0$ and $\rho(y)<0$ for some $y \in L^{2}$, then $(x, y)=\left(y^{+}, y^{-}\right)$prevents $(0,0)$ from being a solution to $(27)$.

Corollary 10 has a "parallel" result in the new framework.

Corollary $15(\rho, \Pi)$ is compatible if and only if $z_{\Pi} \in \Delta_{\rho}$.

Proof. If $(\rho, \Pi)$ is compatible then Corollary $14 f$ implies that $z_{\Pi} \in \Delta_{\rho}$. Conversely, if $z_{\Pi} \in \Delta_{\rho}$ then the proof of the implication $\left.f\right) \Rightarrow a$ ) in Corollary 14 applies again.

Remark 16 Corollary 14 shows that the lack of compatibility often holds. For instance, if $\rho$ is the $C V$ aR then every element in $\Delta_{\rho}$ is essentially bounded (see (21)), and therefore $\rho$ will not be compatible with any pricing model whose SDF is unbounded (Black and Scholes, stochastic volatility models in continuous time, etc.). This result was already pointed out by Balbás et al. (2016a) and others with different proofs. With a similar argument one can show that the weighted $C V a R$ (Rockafellar et al., 2006) and the robust CVaR (Balbás et al., 2016b) are often non compatible with the usual continuous time pricing models of financial economics.

Next let us show that $\tilde{N}(\rho, \Pi)$ may be understood as a "minimum relative (per dollar) price modification" preventing the existence of a $G D$. In order words, let us give a result similar to Corollary 11.

Corollary 17 Consider a solution $\left(\lambda^{*}, \mu^{*}, z^{*}\right)$ of (28), suppose that $\mathbb{P}\left(z^{*}>0\right)=$ 1 , and take $\Pi^{*}(y)=\mathbb{E}\left(y z^{*}\right)$ for every $y \in L^{2}$. Then:

a) $\Pi^{*}(1)=1$.

b) $\tilde{N}\left(\rho, \Pi^{*}\right)=0$. Thus, $\left(\rho, \Pi^{*}\right)$ is compatible.

c)

$$
\tilde{N}(\rho, \Pi) \geq \operatorname{Sup}\left\{\Pi^{*}(x)-\Pi^{*}(y) ; x, y \geq 0, \Pi(x)=\Pi(y)=1\right\},
$$


and the equality holds if (27) is solvable ${ }^{8}$. In particular, $\Pi^{*}=\Pi$ if and only if $(\rho, \Pi)$ is compatible.

d) If $z^{* *} \in L^{2}, \mathbb{E}\left(z^{* *}\right)=1, \mathbb{P}\left(z^{* *}>0\right)=1, \Pi^{* *}(y)=\mathbb{E}\left(y z^{* *}\right)$ for every $y \in L^{2}$, there are no solutions to (28) whose third component is $z^{* *}$, and $\tilde{N}\left(\rho, \Pi^{* *}\right)=0$, then

$$
\tilde{N}(\rho, \Pi) \leq \operatorname{Sup}\left\{\Pi^{* *}(x)-\Pi^{* *}(y) ; x, y \geq 0, \Pi(x)=\Pi(y)=1\right\} .
$$

Proof. a) It trivially follows from (3).

b) If $z^{*}$ replaces $z_{\Pi}$ in (28) then it is obvious that $\left(\lambda^{*}=0, \mu^{*}=1, z^{*}\right)$ becomes (28)-feasible, and therefore $\tilde{N}\left(\rho, \Pi^{*}\right)=0$.

c) As in the proof of Corollary 14, if $\tilde{N}(\rho, \Pi)=0$ then $z^{*}=z_{\Pi}$, and therefore $\Pi^{*}=\Pi$ and the right hand side of (30) equals zero too. Suppose that $\tilde{N}(\rho, \Pi)>0$. Take $x, y \geq 0$ with $\Pi(x)=\Pi(y)=1$. The constraints of $(28)$ imply that

$$
\begin{aligned}
& \mu^{*}-\lambda^{*}=\left(\mu^{*}-\lambda^{*}\right) \mathbb{E}\left(z_{\Pi} y\right) \leq \mathbb{E}\left(z^{*} y\right) \leq \mu^{*} \mathbb{E}\left(z_{\Pi} y\right), \\
& \left(\mu^{*}-\lambda^{*}\right) \mathbb{E}\left(z_{\Pi} x\right) \leq \mathbb{E}\left(z^{*} x\right) \leq \mu^{*} \mathbb{E}\left(z_{\Pi} x\right)=\mu^{*} .
\end{aligned}
$$

Consequently,

$$
\mathbb{E}\left(z^{*} x\right)-\mathbb{E}\left(z^{*} y\right) \leq \mu^{*}-\left(\mu^{*}-\lambda^{*}\right)=\lambda^{*}=\tilde{N}(\rho, \Pi) .
$$

Moreover, if $\left(x^{*}, y^{*}\right)$ solves $(27)$, the second, third, fourth and fifth equalities in (29) lead to (recall that $\lambda^{*}>0$ ):

$$
\begin{aligned}
& \mu^{*}-\lambda^{*}=\left(\mu^{*}-\lambda^{*}\right) \mathbb{E}\left(z_{\Pi} y^{*}\right)=\mathbb{E}\left(z^{*} y^{*}\right), \\
& \mathbb{E}\left(z^{*} x\right)=\mu^{*} \mathbb{E}\left(z_{\Pi} x\right)=\mu^{*}
\end{aligned}
$$

Thus, $\mathbb{E}\left(z^{*} x\right)-\mathbb{E}\left(z^{*} y^{*}\right)=\mu^{*}-\left(\mu^{*}-\lambda^{*}\right)=\lambda^{*}=\tilde{N}(\rho, \Pi)$.

d) Suppose that $\left(\lambda, \mu, z^{* *}\right)$ is never (28)-feasible for $0 \leq \mu-\lambda \leq 1$ and $\mu \geq 1$. Then, for every $\mu \geq 1$ the inequality $z^{* *} \leq \mu z_{\Pi}$ will not hold, because if it held then $\lambda=\mu$ would make $\left(\lambda=\mu, \mu, z^{* *}\right)(28)$-feasible. Thus, for every $\mu \geq 1$ there exists $x_{\mu} \geq 0$ in $L^{2}$ such that $\mathbb{E}\left(z^{* *} x_{\mu}\right)>\mu \mathbb{E}\left(z_{\Pi} x_{\mu}\right)$. Moreover, $\mathbb{E}\left(z_{\Pi} x_{\mu}\right)>0$ due to (25). Replacing $x_{\mu}$ with $x_{\mu} / \mathbb{E}\left(z_{\Pi} x_{\mu}\right)$ if necessary, and still denoting $x_{\mu}$, one can suppose that $\mathbb{E}\left(z_{\Pi} x_{\mu}\right)=1$ and $\mathbb{E}\left(z^{* *} x_{\mu}\right)>\mu$. Taking $y_{\mu}=1$ (riskless security) we have

$$
\mathbb{E}\left(z^{* *} x_{\mu}\right)-\mathbb{E}\left(z^{* *} y_{\mu}\right) \geq \mu-1,
$$

which tends to $+\infty$ as so does $\mu$. Hence, the right hand side of (31) is unbounded and (31) becomes obvious.

Suppose that $\left(\lambda, \mu, z^{* *}\right)$ is (28)-feasible for some $0 \leq \mu-\lambda \leq 1$ and $\mu \geq 1$. Take

$$
\mu^{* *}=\operatorname{Inf}\left\{\mu \geq 1 ; z^{* *} \leq \mu z_{\Pi}\right\},
$$

\footnotetext{
${ }^{8}$ We see that $(27)$ is not necessarily solvable, i.e., it does not necessarily attain its optimal value. This is a difference between Problems (12) and (27) (Proposition 7 and Theorem 19).
} 
and it is obvious that

$$
z^{* *} \leq \mu^{* *} z_{\Pi} .
$$

If $\mu^{* *}=1$ then (33) implies that $z^{* *} \leq z_{\Pi}$, and (3) and (26) will imply that $z^{* *}=z_{\Pi}$. Whence, $\left(\lambda^{* *}=0, \mu^{* *}=1, z^{* *}=z_{\Pi}\right)$ would solve (28), contradicting the assumptions. Thus,

$$
\mu^{* *}>1 .
$$

Take

$$
\lambda^{* *}=\operatorname{Inf}\left\{\lambda ; 0 \leq \mu^{* *}-\lambda \leq 1,\left(\mu^{* *}-\lambda\right) z_{\Pi} \leq z^{* *}\right\} .
$$

The set above is non void because it obviously contains $\lambda=\mu^{* *}$. Furthermore,

$$
0 \leq \mu^{* *}-\lambda^{* *} \leq 1
$$

and

$$
\left(\mu^{* *}-\lambda^{* *}\right) z_{\Pi} \leq z^{* *}
$$

obviously hold. Suppose that $\mu^{* *}-\lambda^{* *}=1$. Then, (36) implies that $z_{\Pi} \leq z^{* *}$, and (3) and (26) imply that $z^{* *}=z_{\Pi}$. Once again we get a contradiction because $\left(\lambda^{* *}=0, \mu^{* *}=1, z^{* *}=z_{\Pi}\right)$ will solve (28), and therefore

$$
0 \leq \mu^{* *}-\lambda^{* *}<1 .
$$

Keeping in mind (34) and (37), we can take $\varepsilon>0$ such that

$$
0 \leq \mu^{* *}-\left(\lambda^{* *}-\varepsilon\right)<1, \quad \mu^{* *}-\varepsilon>1 .
$$

(32) and (35) lead to the existence of $x_{\varepsilon}, y_{\varepsilon} \geq 0$ in $L^{2}$ such that $\mathbb{E}\left(z^{* *} x_{\varepsilon}\right)>$ $\left(\mu^{* *}-\varepsilon\right) \mathbb{E}\left(z_{\Pi} x_{\varepsilon}\right)$ and $\mathbb{E}\left(z^{* *} y_{\varepsilon}\right)<\left(\mu^{* *}-\left(\lambda^{* *}-\varepsilon\right)\right) \mathbb{E}\left(z_{\Pi} y_{\varepsilon}\right)$. Therefore, normalizing so that $\Pi\left(x_{\varepsilon}\right)=\Pi\left(y_{\varepsilon}\right)=1$, and still denoting $x_{\varepsilon}$ and $y_{\varepsilon}$,

$$
\begin{aligned}
& \mathbb{E}\left(z^{* *} x_{\varepsilon}\right)-\mathbb{E}\left(z^{* *} y_{\varepsilon}\right)>\left(\mu^{* *}-\varepsilon\right) \mathbb{E}\left(z_{\Pi} x_{\varepsilon}\right)-\left(\mu^{* *}-\left(\lambda^{* *}-\varepsilon\right)\right) \mathbb{E}\left(z_{\Pi} y_{\varepsilon}\right) \\
& =\left(\mu^{* *}-\varepsilon\right)-\left(\mu^{* *}-\left(\lambda^{* *}-\varepsilon\right)\right)=\lambda^{* *}-2 \varepsilon .
\end{aligned}
$$

Moreover, since (33), (34), (36) and (37) make $\left(\lambda^{* *}, \mu^{* *}, z^{* *}\right)(28)$-feasible, $\lambda^{* *} \geq$ $\lambda^{*}$ must hold, and therefore $\mathbb{E}\left(z^{* *} x_{\varepsilon}\right)-\mathbb{E}\left(z^{* *} y_{\varepsilon}\right)>\lambda^{*}-2 \varepsilon=\tilde{N}(\rho, \Pi)-2 \varepsilon$. If $\varepsilon$ converges to zero we will have (31).

Remark 18 As in Remark 12, one can use the Corollary 17 so as to recover "fair prices". Indeed, if $\Pi^{*}$ replaces $\Pi$ then compatibility will hold, the overpriced marketed claims, characterized by

$$
\Pi^{*}(y)=\mathbb{E}\left(y z^{*}\right)=\left(\mu^{*}-\lambda^{*}\right) \mathbb{E}\left(y z_{\Pi}\right)=\left(\mu^{*}-\lambda^{*}\right) \Pi(y),
$$

(see (29)) will recover a "fair price" once the initial one $\Pi(y)$ is multiplied by $\mu^{*}-\lambda^{*}$, and the under-priced marketed claims, characterized by

$$
\Pi^{*}(x)=\mathbb{E}\left(x z^{*}\right)=\mu^{*} \mathbb{E}\left(x z_{\Pi}\right)=\mu^{*} \Pi(x),
$$

will recover a "fair price" once the initial one $\Pi(x)$ is multiplied by $\mu^{*}$. 


\subsection{Lack of compatibility between CVaR and Black and Scholes or other continuous time pricing models}

Bearing in mind Remark 16, it may be interesting to give the value of $\tilde{N}(\rho, \Pi)$ for some important risk measures and pricing models, which is the purpose of this section.

Along with Assumptions 1 and 3, in this section we also impose Assumption 4 below.

Assumption 4 There does not exists any $(\beta, z) \in \mathbb{R} \times \Delta_{\rho}$ such that $\beta>0$ and $\mathbb{P}\left(\beta z_{\Pi} \leq z\right)=1$.

Assumption 4 frequently holds in practice. For instance, it holds if $z_{\Pi}$ is not essentially bounded (Black and Scholes, stochastic volatility, etc.) and $\Delta_{\rho}$ is composed of essentially bounded random variables $(C V a R$, and very often $R C V a R$ and the weighted $C V a R$, see (20) and (21)). Besides, Assumption 4 enables us to simplify Problem (28).

Theorem 19 a) $(\rho, \Pi)$ is not compatible.

b) Consider the following problem:

$$
\operatorname{Min} \mu\left\{\begin{array}{l}
z \leq \mu z_{\Pi} \\
1 \leq \mu, z \in \Delta_{\rho}
\end{array}\right.
$$

where $(\mu, z) \in \mathbb{R} \times \mathrm{L}^{2}$ is the decision variable. Then, $\left(\lambda^{*}, \mu^{*}, z^{*}\right)$ solves (28) if and only if $\lambda^{*}=\mu^{*}$ and $\left(\mu^{*}, z^{*}\right)$ solves (38). Consequently, (38) is bounded and solvable, and its optimal value equals $\tilde{N}(\rho, \Pi)$.

c) If $\left(\mu^{*}, z^{*}\right)$ solves (38) and $\mathbb{P}\left(z^{*}>0\right)=1$, then Problem (27) is not solvable, although it is bounded and its optimal value is $\tilde{N}(\rho, \Pi)>0$.

d) Suppose that $\alpha \in(0,1),\left(\mu^{*}, z^{*}\right)$ is (38)-feasible and $\rho=C V a R_{\alpha}$. Then, $\left(\mu^{*}, z^{*}\right)$ solves (38) if and only if

$$
z^{*}(\omega)=\operatorname{Min}\left\{\mu^{*} z_{\Pi}(\omega), \frac{1}{1-\alpha}\right\}
$$

out of a $\mathbb{P}-$ null set. Furthermore, $\mathbb{P}\left(z^{*}>0\right)=1$ and therefore Problem (27) is not solvable.

e) Suppose that $\alpha \in(0,1),\left(\mu^{*}, z^{*}\right) \in(1, \infty) \times L^{2}$ and $\rho=C V a R_{\alpha}$. Then, $\left(\mu^{*}, z^{*}\right)$ solves (38) if and only if

$$
\mathbb{E}\left(\operatorname{Min}\left\{\mu^{*} z_{\Pi}, 1 /(1-\alpha)\right\}\right)=1
$$

and (39) holds.

Proof. $a)$ If $(\rho, \Pi)$ were compatible then Corollary 14 shows that $\left(\lambda^{*}, \mu^{*}, z^{*}\right)=$ $\left(0,1, z_{\Pi}\right)$ would solve $(28)$, and therefore it would be (28)-feasible. Thus, $z_{\Pi} \in$ $\Delta_{\rho}$ should hold and $\beta=1$ would contradict Assumption 4 . 
b) If $(\lambda, \mu, z)$ is (28)-feasible, then Assumption 4 trivially implies that $\lambda=\mu$. Then, the equivalence between Problems (28) and (38) becomes straightforward and $b$ ) trivially follows from Theorem 13 .

c) Take the solution $\left(\lambda^{*}, \mu^{*}, z^{*}\right)$ of (28). Statement $a$ ) and Corollary 14 imply that $\mu^{*}>1$, and Assumption 4 and the constraints of (28) imply that $\mu^{*}-\lambda^{*}=$ 0 . If $\left(x^{*}, y^{*}\right)$ solved (27) then (29) would imply $y^{*} z^{*}=0$, and $\mathbb{P}\left(z^{*}>0\right)=1$ would imply $\mathbb{P}\left(y^{*}=0\right)=1$. Notice that $\lambda^{*}=\mu^{*}>1$ and $\mathbb{P}\left(y^{*}=0\right)=1$ contradict the second condition of (29), so (27) cannot be solvable. The rest of the proof trivially follows form Theorem 13 .

d) Suppose that $\left(\mu^{*}, z^{*}\right)$ solves (38). Then $z^{*} \leq \mu^{*} z_{\Pi}$ obviously must hold, and $z^{*} \leq 1 /(1-\alpha)$ holds because $z^{*} \in \Delta_{\rho}\left(\right.$ see $(20)$ with $\left.\frac{d Q}{d \mathbf{P}}=1\right)$. Hence,

$$
z^{*} \leq \operatorname{Min}\left\{\mu^{*} z_{\Pi}, 1 /(1-\alpha)\right\}
$$

Suppose that (41) is not an equality. Then,

$$
1=\mathbb{E}\left(z^{*}\right)<\mathbb{E}\left(\operatorname{Min}\left\{\mu^{*} z_{\Pi}, 1 /(1-\alpha)\right\}\right) .
$$

Consider $\varepsilon>0$ with $\mu^{*}-\varepsilon>1$ (recall that $\mu^{*}>1$ due to $a$ ) and Corollary $14 e$ ) and $\mathbb{E}\left(\operatorname{Min}\left\{\mu^{*} z_{\Pi}, 1 /(1-\alpha)\right\}\right)-\varepsilon>1$. Obviously,

$$
\begin{gathered}
\operatorname{Min}\left\{\mu^{*} z_{\Pi}, 1 /(1-\alpha)\right\}-\operatorname{Min}\left\{\left(\mu^{*}-\varepsilon\right) z_{\Pi}, 1 /(1-\alpha)\right\} \\
\leq \mu^{*} z_{\Pi}-\left(\mu^{*}-\varepsilon\right) z_{\Pi}=\varepsilon z_{\Pi} .
\end{gathered}
$$

Thus, bearing in mind (26),

$$
\mathbb{E}\left(\operatorname{Min}\left\{\left(\mu^{*}-\varepsilon\right) z_{\Pi}, 1 /(1-\alpha)\right\}\right) \geq \mathbb{E}\left(\operatorname{Min}\left\{\mu^{*} z_{\Pi}, 1 /(1-\alpha)\right\}\right)-\varepsilon>1
$$

Since $\mathbb{P}\left(\left(\mu^{*}-\varepsilon\right) z_{\Pi}>0\right)=1$ due to $(25)$,

$$
\mathbb{P}\left(\operatorname{Min}\left\{\left(\mu^{*}-\varepsilon\right) z_{\Pi}, 1 /(1-\alpha)\right\}>0\right)=1
$$

becomes obvious, and $\mathbb{P}\left(\operatorname{Min}\left\{\left(\mu^{*}-\varepsilon\right) z_{\Pi}, 1 /(1-\alpha)\right\} \leq 1 /(1-\alpha)\right)=1$ is obvious too. Thus, (42) leads to

$$
\frac{\operatorname{Min}\left\{\left(\mu^{*}-\varepsilon\right) z_{\Pi}, 1 /(1-\alpha)\right\}}{\mathbb{E}\left(\operatorname{Min}\left\{\left(\mu^{*}-\varepsilon\right) z_{\Pi}, 1 /(1-\alpha)\right\}\right)} \in \Delta_{\rho},
$$

and

$$
\frac{\operatorname{Min}\left\{\left(\mu^{*}-\varepsilon\right) z_{\Pi}, 1 /(1-\alpha)\right\}}{\mathbb{E}\left(\operatorname{Min}\left\{\left(\mu^{*}-\varepsilon\right) z_{\Pi}, 1 /(1-\alpha)\right\}\right)} \leq\left(\mu^{*}-\varepsilon\right) z_{\Pi}
$$

implies that

$$
\left(\mu^{*}-\varepsilon, \frac{\operatorname{Min}\left\{\left(\mu^{*}-\varepsilon\right) z_{\Pi}, 1 /(1-\alpha)\right\}}{\mathbb{E}\left(\operatorname{Min}\left\{\left(\mu^{*}-\varepsilon\right) z_{\Pi}, 1 /(1-\alpha)\right\}\right)}\right)
$$

is (38)-feasible. We have a contradiction because $\left(\mu^{*}, z^{*}\right)$ solves $(38)$. Hence (41) is an equality, and (39) holds.

Conversely, if (39) holds and $\left(\mu^{*}, z^{*}\right)$ does not solve (38) then the solution $(\mu, z)$ of $(38)$ satisfies $\mu<\mu^{*}$, and the proved implication leads to

$$
z=\operatorname{Min}\left\{\mu z_{\Pi}, 1 /(1-\alpha)\right\}
$$


Recalling (3), we have the following chain of relations:

$$
\begin{aligned}
1= & \mathbb{E}\left(z^{*}\right)=\mathbb{E}\left(\operatorname{Min}\left\{\mu^{*} z_{\Pi}, 1 /(1-\alpha)\right\}\right) \\
& \geq \mathbb{E}\left(\operatorname{Min}\left\{\mu z_{\Pi}, 1 /(1-\alpha)\right\}\right) \geq \mathbb{E}(z)=1
\end{aligned}
$$

and therefore

$$
\operatorname{Min}\left\{\mu^{*} z_{\Pi}, 1 /(1-\alpha)\right\}=\operatorname{Min}\left\{\mu z_{\Pi}, 1 /(1-\alpha)\right\} .
$$

Hence, $\mu<\mu^{*}$ and (25) imply that $\mu z_{\Pi} \geq 1 /(1-\alpha)$, and (43) leads to $z=$ $1 /(1-\alpha)$. Therefore, $\mathbb{E}(z)=1 /(1-\alpha)>1$, and we have a contradiction with (3).

e) If (39) and (40) hold then $\left(\mu^{*}, z^{*}\right)$ is (38)-feasible. Therefore, $\left(\mu^{*}, z^{*}\right)$ solves (38) due to $d$ ).

Conversely, suppose that $\left(\mu^{*}, z^{*}\right)$ solves (38). Then, (39) follows from $d$ ). Besides, $z^{*} \in \Delta_{\rho}$ implies that $\mathbb{E}\left(z^{*}\right)=1$, so (39) leads to (40).

Remark 20 Theorem 19 shows how different the indices $\tilde{N}\left(\rho,\left(S_{j}\right)_{j=0}^{m},\left(p_{j}\right)_{j=0}^{m}\right)$ given in Section 3.1 and $\tilde{N}(\rho, \Pi)$ of Section 3.3 are. While

$$
\tilde{N}\left(\rho,\left(S_{j}\right)_{j=0}^{m},\left(p_{j}\right)_{j=0}^{m}\right)
$$

can take any non negative value, and the null value will sometimes hold, $\tilde{N}(\rho, \Pi)$ will be almost always strictly positive (Theorem 19a) and strictly higher than $1=100 \%$ (Theorem 19b). In particular, for $\rho=C V a R_{\alpha}$, keeping in mind Corollary 17 and Theorem 19d, if the pricing rule is modified so as to prevent the existence of a GD, the relative (per dollar) price modification might be larger than $100 \%$ for some marketed claims. Otherwise the existence of a GD could remain true, though it is important to point out that Corollary 17 just provides an upper bound, rather that the exact price relative variation. Anyway, (31) justifies that every substitution of $z_{\Pi}$ must be implemented with a solution of (38) (see also Remark 22 below).

Remark 21 Expressions (39) and (40) significantly facilitate practical computations of $\left(\mu^{*}=\tilde{N}(\rho, \Pi), z^{*}\right)$ if $\rho=C V a R_{\alpha}$. In real examples, and according to Theorem 19d) and 19e), the key condition to estimate $\mu^{*}$ is the equality

$$
\mathbb{E}\left(\operatorname{Min}\left\{\mu^{*} z_{\Pi}, 1 /(1-\alpha)\right\}\right)=1 .
$$

It seems clear that Monte Carlo simulation methods may be useful so as to match (44), though we do not include here any numerical illustrations in order to shorten the exposition.

Remark 22 Let us focus on the Black and Scholes model. Without loss of generality, if one looks for a GD only composed of European style derivatives ${ }^{9}$, then

\footnotetext{
${ }^{9}$ Remark 20 applies for more complex derivatives.
} 
one can simplify the structure of the probability space $(\Omega, \mathcal{F}, \mathbb{P})$. Indeed, assume that $\Omega=(0,1)$ and $\mathbb{P}$ is the Lebesgue measure on the Borel $\sigma$-algebra of this set. The value at $T$ of the underlying asset will have a log-normal distribution given by

$$
S(\omega)=W \operatorname{Exp}\left(\left(r-\frac{\sigma^{2}}{2}\right) T+\sigma \sqrt{T} \Phi^{-1}(\omega)\right),
$$

for $\omega \in(0,1)$, where $W>0$ denotes the current price, and $r$ and $\sigma$ denote the drift and volatility, while $\Phi: \mathbb{R} \longmapsto(0,1)$ is the cumulative distribution function of the standard normal distribution.

This simplification cannot be implemented when pricing path dependent or American style derivatives. In both situations the dynamic evolution of the $G B M$ plays a critical role. Thus, when we choose the simple probability space $(\Omega, \mathcal{F}, \mathbb{P})$ above, we know that we are missing information. However, our simplification is interesting because the exposition is shortened, making it much simpler, and it provides closed formulas for $z^{*}$. We will still obtain solutions of (27) and (28) that will allow the investor to create the sequences of Proposition 1 or satisfying (9). The only restriction is that our sequences will be composed of European style derivatives and might become sub-optimal if more complex securities are involved.

It is known that $z_{\pi}$ is also log-normal and it is the first derivative of the one to one strictly increasing function (Wang, 2000)

$$
(0,1) \ni \omega \leftrightarrow g(\omega)=\Phi\left(\gamma+\Phi^{-1}(\omega)\right) \in(0,1)
$$

where

$$
\gamma=\frac{r}{\sigma} \sqrt{T}
$$

Computing the derivative in (46) we have that

$$
z_{\Pi}(\omega)=\operatorname{Exp}\left(-\frac{\gamma^{2}}{2}-\gamma \Phi^{-1}(\omega)\right)
$$

for $\omega \in(0,1)$, which allows to check easily that $(0,1) \ni \omega \leftrightarrow z_{\Pi}(\omega) \in \mathbb{R}$ is continuous and strictly decreasing. Since it is strictly decreasing and $\mu^{*}>1$, the computation of $\left(\mu^{*}=\tilde{N}(\rho, \Pi), z^{*}\right)$ reduces to the estimation of $p \in(0,1)$ such that (see (39), (40) and (44)):

$$
\frac{p}{1-\alpha}+\frac{1}{(1-\alpha) z_{\Pi}(p)} \int_{p}^{1} z_{\Pi}(\omega) d \omega=1 .
$$

In fact, if one solves (49) then

$$
\mu^{*}=\frac{1}{(1-\alpha) z_{\Pi}(p)}, \quad z^{*}(\omega)=\left\{\begin{array}{ll}
\frac{1}{1-\alpha}, & \omega \leq p \\
\mu^{*} z_{\Pi}(\omega), & \omega \geq p
\end{array} .\right.
$$


In order to solve (49) one can change the variable $\omega=\Phi(u-\gamma)$ in the integral. Straightforward algebra leads to the new equation

$$
\frac{p}{1-\alpha}+\frac{1}{(1-\alpha) z_{\Pi}(p)} \Phi\left(-\gamma-\Phi^{-1}(p)\right)=1,
$$

which may be solved with numerical methods. Solving (50) for the parameters used in Section 3.2, i.e., $\alpha=89.5 \%, r=1 \%, \sigma=60 \%, T=1 / 4$ and (see (47)) $\gamma=0.007900634$, yields a result that satisfies $\mu^{*}>1$. In Section 3.2 we obtained $\tilde{N}\left(\rho,\left(S_{j}\right)_{j=0}^{m},\left(p_{j}\right)_{j=0}^{m}\right) \approx 0.42 \%$. With the same drift, volatility, expiration date, pricing model and risk measure we can obtain $\tilde{N}(\rho, \Pi)=\mu^{*}>100 \%>$ $0.42 \%$. Obviously, the GD size increases because now we are considering every $y \in L^{2}$ as a reachable pay-off, and in Section 3.2 we only dealt with finitely many options. The GD size increases and so does the set of available securities. Nevertheless, the difference

$$
100 \%-0.42 \%=99.58 \%
$$

is really relevant. According to Corollaries 11 and 17, the minimum relative price modification preventing the GD existence might significantly increase as the number of available options tends to infinity, though it is important to point out that Corollary 17 only provides an upper bound, rather that the exact price relative variation. Anyway, (31) and $\mu^{*}>100 \%$ justify that every substitution of $z_{\Pi}$ must be implemented with a solution of (38) (see Remark 20 above).

Remark 23 (Beyond the log-normal distribution) Though Remark 21 yields a general enough estimation method, the simplification of Remark 22 may be interesting when dealing with European style derivatives. In such a case (45) and (48) are not strictly necessary, and the methodology may be extended beyond the Black and Scholes model. Instead of (45), assume that $S_{1}$ is the random value at $T$ of a stochastic pricing process. Suppose that the model has been calibrated and we have chosen a unique $S D F z_{\pi}$ such that (24) applies. Suppose finally that the cumulative distribution function $F:(U, V) \longrightarrow(0,1)$ of the random variable $S_{1}$ is a one to one continuous bijection for some $-\infty \leq U<V \leq \infty^{10}$. Then, the simplification in (45) may be adapted to this new framework, in the sense that one can take

$$
S_{1}(\omega)=F^{-1}(\omega),
$$

where $\omega$ is uniformly distributed on $(0,1)$. Moreover, $z_{\pi}$ may be also understood as a function $(0,1) \ni \omega \longrightarrow z_{\pi}(\omega) \in(0, \infty)$. This setting allows us to easily extend the methodology of Remark 22.

\section{Some Actuarial and Financial Implications}

Many classical problems in finance and insurance deal with risk optimization. This section will be devoted to illustrating how classical problems may become

\footnotetext{
${ }^{10}$ This assumption is not at all restrictive. It holds for many continuous distributions (exponential, normal, log-normal, gamma, Pareto, etc) used in Financial Economics.
} 
unbounded if one faces lack of compatibility. As a consequence, one must recover compatibility before making decisions. Otherwise the problem solution will not make economic sense or lead to wrong decisions.

We will select a few actuarial and financial problems. This is not at all an exhaustive collection of potential applications, but the purpose of this section is just illustrative; One must prevent the existence of a $G D$. Furthermore, for the same reason, we will not present global solutions of the proposed problems, which would require a significantly longer paper.

\subsection{Actuarial examples}

Let us focus on a couple of actuarial classical topics. The first one is the optimal reinsurance problem. Since Borch (1960) and Arrow (1963) proved that, under adequate assumptions, the stop-loss contract minimizes the standard deviation of the ceding company final wealth, this problem has been time and again revisited by many authors. The most recent approaches deal with general risk measures rather than the standard deviation (see, amongst many others, Zhuang et al., 2016, Weng and Zhuang, 2017, etc.), and sometimes also incorporate the effect of the financial market (Guan and Liang, 2014, Peng and Wang, 2016, etc.). Let us point out how the incorporation of the financial market effect may lead to non well-posed optimization problems.

Under the notations of Sections 2 and 3 let us consider that the random variable $u_{0} \in L^{2}$ represents the claims to be paid by a insurer within the time period $[0, T]$. Decompose $u_{0}=u_{r}+u_{c}$, where $u_{r}, u_{c} \in L^{2}$ denote the retained and ceded risk in the reinsurance contract, respectively. If $y \in Y$ is the pay-off provided by the financial market, the insurer final wealth will equal $y-u_{r}$. Thus, if $C>0$ represents the capital to diversify between the financial market and the reinsurance contract, the optimal reinsurance problem may become

$$
\operatorname{Min} \rho\left(y-u_{r}\right)\left\{\begin{array}{l}
\Pi(y)+(1+K) \mathbb{E}\left(u_{0}-u_{r}\right) \leq C, \\
0 \leq u_{r} \leq u, \\
y \in Y, u_{r} \in \mathcal{M},
\end{array}\right.
$$

where $\left(y, u_{r}\right)$ is the decision variable, $K>0$ denotes the loading rate and $\mathcal{M}$ denotes the set of risks $z \in L^{2}$ such that $z$ and $u_{0}-z$ are co-monotone with $u_{0}{ }^{11}$. The objective function of (51) implies that the reinsurer prices according to the "expected value premium principle". This assumption may be significantly relaxed and the rest of the example will remain true, but we present here simply an illustrative example.

Proposition 24 If there is a GD then Problem (51) is unbounded, i.e., there are sequences of feasible decisions whose risk diverges to $-\infty$.

\footnotetext{
${ }^{11}$ Recall that $u_{0}$ and $u_{1}$ are co-monotone if$$
\mathbb{P}\left\{\left[u_{0}\left(\omega_{1}\right)-u_{0}\left(\omega_{2}\right)\right]\left[u_{1}\left(\omega_{1}\right)-u_{2}\left(\omega_{2}\right)\right] \geq 0\right\}=1 .
$$ 
Proof. Consider the sequence $\left(y_{n}\right)_{n=1}^{\infty} \subset Y$ satisfying (9), and take $\left(y_{n}-1\right)_{n=1}^{\infty} \subset$ $Y$ and $u_{r}=u_{0} \in \mathcal{M}$. Then $\left(y_{n}-1, u_{r}\right)$ is feasible because

$$
\Pi\left(y_{n}-1\right)+(1+K) \mathbb{E}\left(u_{0}-u_{r}\right)=0 \leq C .
$$

Moreover, Assumption 1 implies that

$$
\lim _{n \rightarrow \infty} \rho\left(y_{n}-1-u_{r}\right) \leq \rho\left(-1-u_{r}\right)+\operatorname{Lim}_{n \rightarrow \infty} \rho\left(y_{n}\right)=-\infty,
$$

and Problem (51) is unbounded.

Proposition 24 above shows that very important classical actuarial problems do not make sense under the presence of a $G D$. Actually, the provided proof only indicates that the insurer must retain the global actuarial risk because it will be compensated in the financial market ${ }^{12}$.

From a theoretical viewpoint, optimal reinsurance approaches involving both actuarial risk and asset pricing models must deal with $G D$ free models. Otherwise the solution will not exist or will not make sense. A potential solution overcoming this caveat could be to recover the $G D$ absence by modifying the pricing rule according to the lines of Corollary 17.

Approaches involving both actuarial risk and static financial strategies that involve real market quotations also need to fulfill the condition that the in$\operatorname{dex} \tilde{N}\left(\rho,\left(S_{j}\right)_{j=0}^{m},\left(p_{j}\right)_{j=0}^{m}\right)=0$. If it does not hold, then some additional restrictions should be incorporated to the $y$ variable in (51) in order to prevent unbounded solutions. For instance, one could consider an upper bound to the short position value, in line with the constraint imposed in (12). Once these additional restrictions have been incorporated, the existence of a $G D$ will have positive effects on the reached risk level, in the sense that the minimum value of the objective $\rho\left(y-u_{r}\right)$ will decrease. The global fall of $\rho\left(y-u_{r}\right)$ will be closely related to the value of $\tilde{N}\left(\rho,\left(S_{j}\right)_{j=0}^{m},\left(p_{j}\right)_{j=0}^{m}\right)$.

As a second actuarial example, consider an insurer wanting to set the loading rate $K$ to be charged to a set of policies. Denoting by $\left(u_{i}\right)_{i=1}^{N} \subset L^{2}$ the random claims associated with these policies, the global portfolio premium becomes $(1+K) \sum_{i=1}^{N} \mathbb{E}\left(u_{i}\right)$. If this money is invested in the financial market, and $y \in Y$ is the random reached pay-off, the final insurer wealth will become $y-\sum_{i=1}^{N} u_{i}$. Thus, the insurer problem may become

$$
\operatorname{Min} K\left\{\begin{array}{l}
\Pi(y)-(1+K) \sum_{i=1}^{N} \mathbb{E}\left(u_{i}\right) \leq 0, \\
\rho\left(y-\sum_{i=1}^{N} u_{i}\right) \leq 0 \\
y \in Y, K \in \mathbb{R}
\end{array}\right.
$$

where $(K, y)$ is the decision variable. As in Proposition 24, and bearing in mind Proposition 1, if $(\rho, \Pi)$ is non compatible it is easy to see that (52) is unbounded,

\footnotetext{
${ }^{12}$ Notice that the independence between the financial market and the global indemnification did not have to be imposed.
} 
i.e., there are sequences of feasible solutions implying that $K$ diverges to $-\infty$. Hence, the comments about the optimal reinsurance problem apply again. The insurer may modify the pricing rule if a pricing model is used, or he/she must incorporate bounds in the short position value when dealing with real market quotations.

\subsection{Financial Examples}

Since Rockafellar and Uryasev (2000) gave a simple procedure to minimize $C V a R$, many studies in portfolio choice and asset allocation have extended the classical approach of Markowitz with the standard deviation replacing it by an alternative risk measure or robust risk measure ( $V a R, C V a R, R C V a R$, etc.). Amongst the many others, interesting examples are Stoyanov et al. (2007), Haugh and Lo (2001), Dupacová and Kopa (2014), Balbás et al. (2016b), Zhao and Xiao (2016), etc. Expression (9) shows that GD existence implies the availability of sequences of investment strategies whose expected return diverges to $+\infty$ while their risk diverges to $-\infty$. Once again we face unbounded problems and theoretical results without economic sense. In Balbás et al. (2016b) the authors propose to enlarge the ambiguity level of the investor. Alternatively, for non ambiguous agents one could deal with the ideas of this paper. Corollaries 11 and 17 propose ways to modify the pricing rules, while Problems (12) and (27) propose solutions to make the problems bounded. Anyway, according to the empirical analysis yielded by Balbás et al. (2016a), the intuition is that

high values of $\tilde{N}\left(\rho,\left(S_{j}\right)_{j=0}^{m},\left(p_{j}\right)_{j=0}^{m}\right)$ or of $\tilde{N}(\rho, \Pi)$ will imply that the investor might be able to create strategies with a very attractive return/risk ratio.

Many more classical financial problems may be treated with risk measures. For instance, pricing and hedging issues (Goovaerts and Laeven, 2008, Balbás et al., 2010), risk management (Ahn et al., 1999, Constantinides et al., 2011), regulatory capital etc. All of these often lead to unrealistic solutions in the presence of a $G D$, which implies that the pricing rules will have to be changed. If there are no pricing processes involved, and only market quotations are being considered, appropriate bounds must be imposed. Both, constraints in line with Problems (12) and (12), and constraints related to the limit order book will have to be considered. It may be important to point out that the additional constraints of Problems (12) and (12) will be quite similar to those related to the restrictions of the limit order book.

\section{Conclusion}

The existence of a $G D$ is anti-intuitive and should not make any economic sense, but it often holds in practice. The $G D$ size has been measured for both real market quotations and theoretical pricing models. In both cases the provided index has optimized the strategy risk with respect to the value of the sold assets, which means that we are measuring in monetary and relative terms. 
If only real market quotations are involved and the risk measure is robust then the approach is also model-independent. In this case the $G D$ measure has a dual interpretation in terms of the minimum relative (per dollar) price modification preventing the $G D$ existence. Moreover, it yields information to identify which are the over-priced and the under-priced securities. Numerical examples in the paper illustrate these cases.

If a pricing model is involved then the dual interpretation above still applies, as well as the comments about over or under priced pay-offs, but there are also very important differences with respect to the model-independent case. Firstly, if the $S D F$ is not essentially bounded then $G D$ existence will always hold if the risk measure sub-gradient is composed of essentially bounded random variables. Secondly, the $G D$ size (i.e., the index value) will be much higher. Actually, it will be higher than $100 \%$, while this value is quite difficult to reach with a finite collection of real market data. Explicit formulas of the $G D$ size are given here for the Black and Scholes model.

Lastly, it is important to remark that the $G D$ existence may provoke pathologies in many classical actuarial and financial problems. Concrete examples have been provided. The developed methodology allows the agent to overcome these pathologies and prevent wrong decisions.

\section{Acknowledgments}

The authors thank the anonymous reviewers for their useful suggestions. This research partially supported by the Natural Sciences and Engineering Research Council (NSERC) of Canada, grant 36860-2012 and was completed while the 2nd author visited the University of California at Santa Barbara. The usual caveat applies.

\section{References}

Ahn, D., J. Boudoukh, M. Richardson and R.F. Whitelaw (1999). Optimal risk management using options. The Journal of Finance, 54, 359 - 375.

Anderson, E.J. and P. Nash (1987). Linear programming in infinite-dimensional spaces. John Wiley \& Sons.

Arrow, K.J. (1963). Uncertainty and the welfare of medical care. American. Economic. Review, 53, 941-973.

Artzner, P., F. Delbaen, J.M. Eber and D. Heath (1999). Coherent measures of risk. Mathematical Finance, 9, 203 - 228.

Balbás, A., B. Balbás and R. Balbás (2016a). Outperforming benchmarks with their derivatives: Theory and empirical evidence. The Journal of Risk, 18, $4,25-52$.

Balbás, A., B. Balbás and R. Balbás (2016b). Good deals and benchmarks in robust portfolio selection. European Journal of Operational Research, 250, $666-678$.

Balbás, A. and R. Balbás (2009). Compatibility between pricing rules and 
risk measures: The $C C V a R$. Revista de la Real Academia de Ciencias, RACSAM, 103, $251-264$.

Balbás, A., R. Balbás and J. Garrido (2010). Extending pricing rules with general risk functions. European Journal of Operational Research, 201, 23 - 33.

Balbás A., I.R. Longarela and J. Lucia (1999). How financial theory applies to catastrophe-linked derivatives. An empirical test of several pricing models. Journal of Risk and Insurance, 66, 4, 551 - 582.

Balbás A., I.R. Longarela and A. Pardo (2000). Integration and arbitrage in the Spanish financial markets: An empirical approach. Journal of Futures Markets, 20, 4, 321 - 344.

Ballestero, E., M. Bravo, B. Perez-Gladish, M. Arenas-Parra and D. PlaSantamaria (2012). Socially Responsible Investment: A multicriteria approach to portfolio selection combining ethical and financial objectives. European Journal of Operational Research, 216, 487 - 494.

Ballestero, E., and C. Romero (1996). Portfolio Selection: A Compromise Programming Solution. Journal of the Operational Research Society, 47, 1377 - 1386.

Bondarenko, O. (2014). Why are put options so expensive? Quarterly Journal of Finance, 4, 3, 1450015.

Borch, K. (1960). An attempt to determine the optimum amount of stop loss reinsurance. In: Transactions of the 16th International Congress of Actuaries I, 597-610.

Chen, Z., and P.J. Knez (1995). Measurement of market integration and arbitrage. The Review of Financial Studies, 8, 2, 287 - 325.

Constantinides, G.M., M. Czerwonko, J.C. Jackwerth and S. Perrakis (2011). Are options on index futures profitable for risk-averse investors? Empirical evidence. The Journal of Finance, 66, 1407-1437.

Dash, G.H., and N. Kajiji (2014). On multiobjective combinatorial optimization and dynamic interim hedging of efficient portfolios. International Transactions in Operational Research, 21, 899 - 918.

Davis M.H.A., V.G. Panas and T. Zariphopoulou (1993). European option pricing with transaction costs. Siam Journal of Control and Optimization, 31, $470-493$.

Duffie, D. (1988). Security markets: Stochastic models. Academic Press.

Dupacová, J. and M. Kopa (2014). Robustness of optimal portfolios under risk and stochastic dominance constraints. European Journal of Operational Research, 234, 434 - 441.

Goovaerts, M.J. and R. Laeven (2008). Actuarial risk measures for financial derivative pricing. Insurance: Mathematics and Economics, 42, 540-547.

Guan, G. and Z. Liang (2014). Optimal reinsurance and investment strategies for insurer under interest rate and inflation risks. Insurance: Mathematics and Economics, 55, $105-115$.

Harrison, J. and D. Kreps (1979). Martingales and arbitrage in multiperiod securities markets. Journal of Economic Theory, 20, 381 - 408.

Haugh, M.B. and A.W. Lo (2001). Asset allocation and derivatives. Quantitative Finance, 1, 45-72. 
Kamara, A., and T.W. Miller (1995). Daily and intradaily tests of European put-call parity. Journal of Financial and Quantitative Analysis, 30, 4, 519 - 541.

Kempf, A., and O. Korn (1998). Trading system and market integration. Journal of Financial Intermediation, 7, 220 - 239.

Luenberger, D.G. (2001). Projection pricing. Journal of Optimization Theory and Applications, 109, 1 - 25.

Peng, X. and W. Wang (2016). Optimal investment and risk control for an insurer under inside information. Insurance: Mathematics and Economics, 69, $104-116$.

Prisman, E.Z. (1986). Valuation of risky assets in arbitrage free economies with frictions. The Journal of Finance, 41, 3, 545 - 556.

Rockafellar, R. T. and S. Uryasev (2000). Optimization of conditional-valueat-risk. The Journal of Risk, 2, 21 - 42.

Rockafellar, R. T., S. Uryasev and M. Zabarankin (2006). Generalized deviations in risk analysis. Finance and Stochastics, 10, 51 - 74.

Sawaragi, Y., H. Nakayama and T. Tanino (1985). Theory of Multiobjective Optimization. Elsevier,

Schaeffer, H.H. (1970). Topological vector spaces. Springer.

Stoyanov, S.V., S.T. Rachev and F.J. Fabozzi (2007). Optimal financial portfolios. Applied Mathematical Finance, 14, 401-436.

Wang, S.S. (2000). A class of distortion operators for pricing financial and insurance risks. Journal of Risk and Insurance, 67, 15-36.

Weng, C. and S.C. Zhuang (2017). CDF formulation for solving an optimal reinsurance problem. Scandinavian Actuarial Journal, forthcoming, DOI: 10.1080/03461238.2016.1167114.

Zhao, P. and Q. Xiao (2016). Portfolio selection problem with Value-at-Risk constraints under non-extensive statistical mechanics. Journal of Computational and Applied Mathematics, 298, 74-91.

Zhuang, S.C., C. Weng, K.S. Tan and H. Assa (2016). Marginal indemnification function formulation for optimal reinsurance. Insurance: Mathematics and Economics, 67, 65-76. 